
\title{
25 Research Square \\ Invadopodia enable cooperative invasion and metastasis of breast cancer cells
}

\section{Louisiane Perrin}

Temple University

\section{Battuya Bayarmagnai}

Temple University

Erkan Tuzel

Temple University

Bojana Gligorijevic ( $\square$ bojana.gligorijevic@temple.edu )

Temple University https://orcid.org/0000-0001-9071-7467

\section{Article}

Keywords: breast cancer, metastasis, heterogeneous tumors, collective invasion, dissemination

Posted Date: June 25th, 2021

DOI: https://doi.org/10.21203/rs.3.rs-632734/v1

License: (c) (1) This work is licensed under a Creative Commons Attribution 4.0 International License. Read Full License

Version of Record: A version of this preprint was published at Communications Biology on August 1st, 2022. See the published version at https://doi.org/10.1038/s42003-022-03642-z. 


\section{Abstract}

Multiple clones of cancer cells can seed metastases via collective invasion and dissemination. While it is known that cancer clones can cooperate during invasion, the events leading to it and the effects it may have on metastasis are not known. In this study, we demonstrate that, when mixed in 3D spheroids, the isogenic 4T1 and 67NR breast cancer cells sort from each other, followed by their cooperative invasion. By time-lapse microscopy of heterogenous spheroids embedded in collagen I, we show that the invasive $4 \mathrm{~T} 1$ cells that reach the spheroid edge remain there, while the non-invasive 67NR cells move randomly. This results in cell sorting and enrichment of invasive $4 \mathrm{~T} 1$ cells at the spheroid periphery. Following cell sorting, 4T1 cells lead the 67NR cells in cooperative invasion. Elimination of invadopodia in 4T1 cells, by knockdown of the protein Tks5, blocks invasion and demonstrates that invasion requires invadopodia only in leader cells and not in follower cells. Importantly, using syngeneic mouse model, we demonstrate that cells with and without invadopodia can also engage in cooperative metastasis. Altogether, our results suggest that a few clones with invadopodia could drive the metastasis of cell clusters from heterogeneous tumors.

\section{Introduction}

More than $90 \%$ of cancer patients die due to complications resulting from metastasis, i.e. the process of dissemination, re-seeding and growth of cancer cells in secondary organs $(1,2)$. In breast cancer, recent work demonstrated that metastases mostly arise from polyclonal seedings $(3,4)$. These polyclonal metastases develop from the collective dissemination of cell clusters, as opposed to the successive accumulation of multiple single clones. Together with the growing literature on phenotypic heterogeneity within primary tumors (5), these observations suggest that cooperativity between clones of cancer cells may facilitate metastasis.

In breast cancer, the metastatic cascade is initiated when cancer cells acquire invasive properties, which includes the integration of motility and the ability to degrade the extracellular matrix (ECM) (2). Both invasion and motility are commonly associated with the activation of the epithelial-mesenchymal transition (EMT) program (6). During EMT, epithelial cells gradually lose cell-cell contacts, progressively strengthen their adhesions to the ECM and increase their contractility, becoming motile. Concomitantly to EMT, cancer cells can also acquire the capacity to locally degrade the ECM using invadopodia (7-10). Invadopodia are membrane protrusions enriched in matrix metalloproteinases (MMPs) that confer cancer cells with high proteolytic activity $(11,12)$ and importantly, elevated metastatic potential $(13,14)$. Since the EMT program is not a binary, nor a unidirectional switch, and since multiple EMT routes exist, distinct EMT trajectories may result in cancer clones with different levels of invasiveness (15).

Recent 3D in vitro work on breast cancer showed that invasive collective strands are composed of cancer cells that differ in multiple invasive traits $(3,16-20)$. For example, compared to follower cells, leader cells show increased contractility $(19)$, cell-ECM adhesion $(3,16)$, ECM remodeling $(20)$ and ECM degradation capacities $(18,19)$. As a result, leader cells can enable the invasion of otherwise non-invasive follower 
cells, a phenomenon termed cooperative invasion (21). Our recent work showed that leader cells largely reside in the $\mathrm{G} 1$ phase of the cell cycle, the phase of the cell cycle during which invadopodia-mediated ECM degradation is the highest (22). These data suggest that during collective invasion, leader cells may preferentially assemble invadopodia. Although breakdown of the ECM is clearly required for collective invasion, the role of invadopodia-mediated ECM degradation in leader $v s$. follower cells is unclear. To this date, none of the studies detailed the spatial reorganization that may precede the cooperative invasion. Furthermore, as all the previous studies investigated cooperative invasion in 3D cultures, the cooperation during dissemination and metastasis were not yet explored.

In this study, we aim to understand how cancer clones with differential invasive skills may cooperate during invasion and metastasis. We show that invasive clones can sort from and lead non-invasive clones into cooperative invasion and metastasis. Our study suggests that cooperativity between cancer cells may be an efficient mechanism for collective metastasis.

\section{Results}

\section{T1 cells, but not $67 \mathrm{NR}$ cells, invade into collagen I}

To investigate how cancer clones with differential invasive skills cooperate during metastasis we used the isogenic pair of breast cancer cell lines $4 \mathrm{~T} 1$ and $67 \mathrm{NR}$, syngeneic to Balb/C mice $(23,24)$. Upon orthotopic implantation, both cell lines grow primary tumors, but only 4T1 cells metastasize (25). We assessed the invasive capacities of 4T1 and 67NR cells in the spheroid invasion assay in the high-density collagen I, which requires MMP-driven degradation of the matrix (22). After two days, we found that the 4T1 cells exhibited robust invasion into the collagen I matrix, while 67NR cells did not invade (Fig. 1A, B). Treatment with the pan-MMP inhibitor GM6001 effectively blocked invasion of 4T1 cells (Fig. 1A, B). In addition, immunofluorescence labeling of MMP-mediated collagen I cleavage sites (Col 3/4) showed that the invasion of $4 \mathrm{~T} 1$ cells into the matrix was MMP-dependent (Fig. 1C). Treatment with mitomycin C, which impairs cell division (26), confirmed that the invasion of $4 \mathrm{~T} 1$ cells was not due to cell proliferation (Fig. 1A, B and Fig. S1). 4T1 cells are known to invade as collective strands, with presence of E-cadherin at cell-cell junctions $(27,28)$. We confirmed that $4 \mathrm{~T} 1$ cells expressed E-cadherin, while $67 \mathrm{NR}$ cells expressed N-cadherin (Fig. S2A, B) (27-29). Both 4T1 and 67NR cell lines expressed vimentin (Fig. S2A). Interestingly, on the EMT axis (phenotypic continuum from epithelial to mesenchymal), this classifies the invasive $4 \mathrm{~T} 1$ cells as epithelial/mesenchymal and the non-invasive 67NR cells as mesenchymal. Further, in the $4 \mathrm{~T} 1$ spheroids, we found E-cadherin to be enriched at the junctions between the leader and follower cells, which verified that the integrity of E-cadherin-mediated cell-cell junctions was maintained during invasion (Fig. S2C, D) (30). Overall, these results demonstrated that 4T1 cells perform MMP-dependent collective invasion, while the 67NR cells do not invade into dense collagen I matrix.

Invadopodia are membrane protrusions enriched in actin, actin-binding proteins, such as cortactin and Tks5, and MMPs $(11,12)$. As invadopodia function results in local ECM degradation, we hypothesized that invadopodia play a role in the invasion of $4 \mathrm{~T} 1$ cells. We also reasoned that the observed difference in 
the invasion capacities of $4 \mathrm{~T} 1 \mathrm{vs}$. 67NR cells might be explained, at least in part, by a disparity in their invadopodia function. To test this, we first analyzed the expression level of key invadopodia components cortactin and Tks5 (11). Both cell lines expressed similar levels of cortactin and Tks5 (Fig. 1D). We next measured invadopodia function by culturing cells on top of fluorescently labeled gelatin, which allows visualization of degradation as holes in the matrix (31). We found that $4 \mathrm{~T} 1$ cells, but not $67 \mathrm{NR}$ cells, were able to degrade the gelatin layer (Fig. 1E, F). Puncta of co-localized Tks5, F-actin and degraded gelatin, indicative of functional and mature invadopodia, were present in 4T1 cells (Fig. 1G). This suggests that the observed degradation holes were generated by invadopodia. To examine whether invadopodia also play a role in the invasion of 4T1 cells in spheroids, we labeled spheroids for F-actin, Tks5 and MMPmediated collagen I cleavage sites. We identified functional invadopodia in leader cells, demonstrated by co-localization of F-actin, Tks5 and MMP-mediated collagen I cleavage sites (Fig. 1H). These observations established a link between invadopodia and collective invasion of 4T1 cells and suggest that leader cells assemble invadopodia.

Since the invasion phenotype consists of invadopodia-mediated ECM degradation and cell migration (32), we performed a scratch assay to investigate whether 67NR cells can migrate as efficiently as 4T1 cells. We tracked individual cells (Fig. 1I, J and Movie S1) and found that while the instantaneous speed of $67 \mathrm{NR}$ cells is higher than that of $4 \mathrm{~T} 1$ cells (Fig. 1K), 4T1 cells are significantly more persistent than 67NR cells (Fig. 1L).

In summary, we showed that the 4T1/67NR pair is a suitable tool to investigate how cancer clones with differential invasive capacities cooperate during invasion.

\section{Persistence drives cell sorting between 4T1 and 67NR cells}

We next set out to investigate dynamics of spatial organization and invasion in the spheroids where 4T1 and 67NR are mixed together. While self-organization and patterning of cells is heavily studied in embryonic tissues and during development, so far, only a few studies tested mixtures of two or more cell types in the cancer spheroid model $(19,20)$. Most studies reported that cells sort based on the differential adhesion hypothesis, which predicts sorting based on differences in the intercellular adhesiveness, with cells that exhibit the strongest cell-cell adhesions positioned in the center (33). Based on the expression of cadherins for the 4T1/67NR pair, we reasoned that in a 3D spheroid, 4T1 will sort from 67NR cells, with $\mathrm{N}$-cadherin-expressing 67NR cells surrounding E-cadherin-expressing 4T1 cells.

To test our hypothesis, we generated 67NR-GFP and 4T1-mScarlet cell lines. These cells were used to form mixed spheroids at a 1:50 ratio of 4T1-mScarlet:67NR-GFP cells, to account for the difference in their proliferation rate over the course of the spheroid invasion assay (Fig. S3A, B). We next embedded the mixed spheroids in collagen I and performed longitudinal imaging, i.e. we imaged a given spheroid daily (Fig. 2A and Fig. S3C) (34). We noticed that 67NR and 4T1 cells sorted from each other starting on day 3. Individual optical slices (Movie S2) and the analysis of cell coordinates clearly demonstrated the enrichment of $4 \mathrm{~T} 1$ cells at the edge of spheroids (Fig. 2B and Fig. S3D). To quantify cell sorting, we calculated the relative distance of each cell to the spheroid center, a metric we named Distance Index (DI), 
such that a value of 0 marks a cell at the spheroid center and a value of 1 corresponds to a cell at the spheroid-collagen I interface (Fig. 2C). At day 3 after embedding the spheroids in collagen I, we found that the DI of 4T1 cells increased over time and was significantly higher than the DI of 67NR cells (Fig. 2D). This was also observed for an individual spheroid (Fig. S3E). These data revealed that, over the course of 3 days, cells reorganized from a random distribution to spheroids with 4T1 cells populating the interface and 67 NR cells located in the spheroid core. On days 4-6, cell sorting of 4T1 and 67NR cells was followed by the invasion (Fig. S3F).

Spheroids of 4T1 cells have been shown to contain laminin, collagen I and fibronectin in the extracellular space (35). The presence of ECM within a spheroid suggests that 3D cell motility, and consequently cell sorting, may require MMPs. To test the link between motility, MMPs and cell sorting, we treated spheroids with an inhibitor of cell contractility (ROCK inhibitor, Y-27632) or a pan-MMP inhibitor, GM6001. We found that both treatments blocked cell sorting (Fig. 2E, F). We then performed time-lapse imaging of mixed spheroids. We tracked individual cells within the spheroid (Movies S3, 4) and for each cell, calculated the difference between the initial and final distance indices (DDI=DI for the last position of a given cell - DI for the initial position of a given cell), such that a positive DDI indicates cell motility towards the spheroid edge ("out" in Fig. 2G,I), and a negative DDI indicates movement towards the spheroid center ("in" in Fig. 2G-I). A null DDI indicates no net movement. We once again classified each cell based on their initial position as edge or core, and defined the edge compartment as a two-cell layer closest to the spheroidcollagen interface (30 $\mu$ m-wide elliptical ring; Fig. $2 \mathrm{G})$. We found that the percentage of tracked cells that switched between compartments during the duration of the time-lapse imaging was negligible for all cells, except for the 4T1 cells initially located in the core (Fig. S4A). For 4T1 cells, we found that the DDI was significantly higher for cells whose initial position was in the spheroid core compared to cells whose initial position was at the spheroid edge (Fig. $2 \mathrm{H}, \mathrm{I})$. This indicates that $4 \mathrm{~T} 1$ cells moved from the spheroid core towards the spheroid edge. In contrast, $4 \mathrm{~T} 1$ cells located at the spheroid edge had a DDI close to zero, suggesting that $4 \mathrm{~T} 1$ cells that initially found at the spheroid edge moved within that compartment only (Fig. 2I). The DDIs were minimal for all 67NR cells, regardless of their initial position, indicating that these cells moved only within the compartments in which they were initially located (Fig. 2I). Since the DDI compares the initial and the final positions of cells, this metric does not provide information on the persistence of cells. Indeed, for a given DDI, the cell trajectory may be more or less tortuous. By computing the mean square displacement (MSD) of cells in the polar coordinate system, which captures spheroid symmetry, we determined that $4 \mathrm{~T} 1$ cells are more persistent in the radial direction (super-diffusive, $a>1$ ) than 67NR cells (diffusive, $a=1$ ) (Fig. 2J, K and Fig. S4B-D). Both 4T1 and 67NR cells had similar motility behavior in the angular direction (Fig. 2J and Fig. S4B, C). Treatment of spheroids with GM6001 impaired the movement of 4T1 cells from the core to the edge (Fig. 2I-K), and resulted in a reduced effective diffusion coefficient of all cells in the radial direction (Fig. 2J, K; Fig. S4B-D and Movies S5, 6). We confirmed that the spheroid growth was similar in the GM6001 and DMSO conditions, suggesting that the loss of cell sorting was independent from cell proliferation (Fig. S4E). Taken together, these results indicate that cell sorting within mixed spheroids is driven primarily by the directed motility of $4 \mathrm{~T} 1$ cells from the core to the edge compartment and their ability to remain at the 
edge, while 67NR cells exhibit random (diffusive) motility, remaining in the same compartment over time. In summary, we showed that differences in persistence drive cell sorting between 4T1 and 67NR cells.

\section{An adhesive ECM interface is required for cell sorting}

Since cell sorting occurred by day 3 post collagen-embedding (Fig. 2A, D), and the accumulation of 4T1 cells at the spheroid edge was maintained throughout 3D invasion (Fig. 2I), we reasoned that the interaction of $4 \mathrm{~T} 1$ cells with the ECM may be critical for maintaining cell sorting. To test this, we placed mixed spheroids in a non-adherent matrix composed of agarose. At day 3 and 4 , the DI was similar for both $4 \mathrm{~T} 1$ cells and 67NR cells, demonstrating that cells embedded in agarose did not sort (Fig. 3A, B). The area of spheroid core was similar in both collagen I and agarose matrices, indicating that the loss of cell sorting was independent from cell proliferation (Fig. S5A). This absence of cell sorting could be due to the failure of $4 \mathrm{~T} 1$ cells to remain at the spheroid edge without the adhesive ECM. To test this in realtime, we performed time-lapse imaging of mixed spheroids embedded in agarose and tracked individual 4T1 cells (Fig. 3C; Movie S7). We found that the average DDI for cells initially located at the edge compartment was negative, and close to zero for cells initially located in the core compartment (Fig. 3D). This indicates that 4T1 cells move from the spheroid edge compartment into the core compartment (Movie S8), and within the core (Movie S7). Those 4T1 cells which enter the edge compartment subsequently leave it, which was not observed in spheroids embedded in collagen I matrix (Movie S9). Interestingly, by computing the MSD of cells in the radial direction, we found that the difference in persistence between 4T1 (super-diffusive, $a>1$ ) and 67NR (diffusive, $a=1$ ) cells was maintained (Fig. 3E and Fig. S5B-D), suggesting that an adhesive ECM interface is required for cell sorting.

This prompted us to hypothesize that, compared to $67 \mathrm{NR}$ cells, $4 \mathrm{~T} 1$ cells preferentially adhere to the ECM. To compare the adhesive properties of $4 \mathrm{~T} 1$ and $67 \mathrm{NR}$ cells to ECM, we developed a 2D cell-ECM adhesive competition assay, in which both cell types were plated on top of circular gelatin islands $(5.5 \mathrm{~mm}$ in diameter), with poly-L-lysine coating present between the islands (Fig. 3F). After $24 \mathrm{~h}$, we scored the number of $4 \mathrm{~T} 1$ and $67 \mathrm{NR}$ cells that migrated from the gelatin islands onto the poly-L-lysine coated region. We found that $85 \%$ of the cells present on the poly-L-lysine region at $24 \mathrm{~h}$ timepoint were $67 \mathrm{NR}$ cells (Fig. 3G). To explain this, we analyzed the time-lapse movies, and saw that all 4T1 cells that crossed from the gelatin onto the poly-L-lysine area migrated back to the gelatin (Movie S10). We wondered whether this could be explained by $4 \mathrm{~T} 1$ cells having a higher adhesion strength to gelatin than 67NR cells. To test this, we measured the contact angle of cells plated on gelatin or poly-L-lysine (Fig. $3 \mathrm{H})$. The cell-matrix contact angle was previously shown to increase with the adhesion strength (36). We found that both cell types had similar contact angle when plated on gelatin (Fig. 3I). However, 4T1 cells plated on poly-L-lysine had a significantly lower contact angle, while 67NR cells exhibited similar contact angle values on both gelatin and poly-L-lysine (Fig. 3I), indicating that, compared to 67NR cells, 4T1 cells are more sensitive to the presence of ECM. This affinity to ECM is in line with the observation that the FAK protein level is higher in $4 \mathrm{~T} 1$ cells than in 67NR cells (Fig. S5E). By analyzing cell-cell contacts between $4 \mathrm{~T} 1$ and $67 \mathrm{NR}$ cells plated on gelatin, we found that the percentage of homotypic contacts was similar at the time of plating $(0 \mathrm{~h})$ and at $24 \mathrm{~h}$ post plating (Fig. S5F, G). This confirmed that $4 \mathrm{~T} 1$ and $67 \mathrm{NR}$ cells did 
not sort in 2D, suggesting that the differential adhesion is not the main driver for cell sorting in our system and emphasizing the requirement for a cell-ECM interface to initiate cell sorting (33).

\section{T1 cells lead 67NR cells in an MMP-dependent cooperative invasion}

Following sorting of $4 \mathrm{TT} 1$ and $67 \mathrm{NR}$ cells in spheroids at day 3 and 4, invasion occurred at day 5 and 6 (Fig. 2A and Fig. 4A; Fig. S3C). One mechanism by which cancer cells can invade collectively is cooperative invasion: invasive leader cells create microtracks inside the ECM, through which non-invasive cells can follow (18-21). Since 4T1 and 67NR cells display differential invasive skills, we reasoned that 4T1 cells could enable the cooperative invasion of the non-invasive 67NR cells. To test this hypothesis, we analyzed the mixed spheroids at day 6 post embedding. We observed that the non-invasive 67NR cells present in the mixed spheroids entered the collagen I matrix (Fig. 4A). Accordingly, we found that, compared to 67NR-only spheroids, mixed spheroids had a higher number of strands per spheroid (Fig. 4B), and approximately one third of the strands contained 67NR cells (Fig. 4C). Importantly, in mixed spheroids, the majority of strands was led by $4 T 1$ cells (Fig. 4D). In line with the MMP-dependency of 4T1 cells for invasion (Fig. 1A), GM6001 blocked the mixed spheroid invasion (Fig. 4A-C). During cooperative invasion, cell sorting between $4 \mathrm{~T} 1$ and $67 \mathrm{NR}$ cells was maintained in the invasive strands as well as within the spheroid (Fig. 4E-G), with the 4T1 cells lining the spheroid-matrix interface. Overall, these findings demonstrate that cooperative invasion of mixed spheroids into collagen I is MMP-dependent, with $4 \mathrm{~T} 1$ cells assuming the leader position.

Our results so far suggest that the 4T1-mediated ECM degradation is required for 67NR cells to enter the collagen I matrix. We hypothesized that 4T1 cells presence in the collagen I matrix is necessary and sufficient to degrade the collagen and create microtracks. To confirm this, instead of pre-mixing 4T1 cells with 67NR cells in a spheroid, we embedded 67NR spheroids in collagen I matrix populated by 4T1 cells. Similar to our observations in mixed spheroids, we found that in the presence of $4 \mathrm{~T} 1$ cells in the collagen I matrix, 67NR cells invaded into the matrix, and that this invasion was MMP-dependent (Fig. S6A, B). To exclude the possibility that soluble MMPs released by $4 \mathrm{~T} 1$ cells facilitated the invasion of $67 \mathrm{NR}$ cells into the collagen I matrix, we cultured spheroids of 67 NR cells with conditioned medium from 4T1 cells. We found that providing conditioned medium from 4T1 cells to 67NR cells was not sufficient for 67 NR cells to invade into the collagen I matrix (Fig. S6C, D). In mixed spheroids, we identified microtracks inside the collagen I matrix, filled with 4T1 cells leading and 67NR cells following (Fig. S6E). Previous report of a heterotypic cooperative invasion between cancer-associated fibroblasts and epithelial cancer cells has demonstrated involvement of heterotypic $\mathrm{N}$-cadherin/E-cadherin adhesions (37). We did not detect heterotypic E-cadherin/N-cadherin junctions between 4T1 and 67NR cells (Fig. S7A-C), suggesting that the cooperative invasion between these cells was not dependent on $\mathrm{E}-$ / $\mathrm{N}$-cadherin interactions. Overall, our data suggest that 4T1 cells degrade the collagen I matrix and 67NR cells move into the microtracks created by $4 \mathrm{~T} 1$ cells.

\section{Cells with invadopodia lead cells without invadopodia in cooperative invasion}


Given that 4T1-mediated ECM degradation is required for cooperative invasion, we wondered whether specifically invadopodia assembly by $4 \mathrm{~T} 1$ cells was responsible for ECM degradation. To rigorously confirm that invadopodia function is required for cooperative invasion of cancer cells, we stably eliminated invadopodia in 4T1-mScarlet cells, using a knockdown of Tks5 (4T1-KD) (13), and established the corresponding 4T1-control (4T1-CTL) cell line (Fig. 5A). We confirmed by gelatin degradation assay in $2 \mathrm{D}$ and spheroid invasion assay in 3D that 4T1-KD cells lost their invasive capacity (Fig. 5B-E). We also verified that 4T1-CTL and 4T1-KD cell lines still expressed E-cadherin (Fig. 5A). We then performed the spheroid invasion assay of mixed spheroid containing 4T1-CTL and 4T1-KD cells. At 2 days after embedding, we found that, in addition to the 4T1-CTL cells, the non-invasive 4T1-KD cells had entered the collagen I matrix (Fig. 5D). Compared to 4T1-KD spheroids, mixed spheroids had an increased number of strands (Fig. 5E), most of which contained 4T1-KD cells (Fig. 5F) and were led by 4T1-CTL cells (Fig. 5G). Similar results were obtained when invadopodia were eliminated in the human breast cancer cell line MDA-MB-231 (Fig. S8A-G). To further test the importance of invadopodia in cooperative invasion of cancer cells, we performed the spheroid invasion assay for a mixture of 4T1-KD and 67NR-GFP cells. As expected for a mixture of clones without invadopodia, we did not observe invasive strands (Fig. 5H, I). Overall, these results demonstrate that functional invadopodia are required in leader cells during cooperative invasion, while follower cells can lack invadopodia.

We also tested if 4T1-KD cells demonstrated sorting when mixed with 4T1-CTL or 67NR. As expected, cell sorting did not occur in mixed spheroids of 4T1-CTL and 4T1-KD cells (Fig. S9A), but it did occur in spheroids containing 4T1-KD and 67NR-GFP cells (Fig. S9B). These findings confirmed that invadopodia are not required for cell sorting. Since 4T1-CTL and 4T1-KD cells display similar motility and cell-ECM adhesion properties (Fig. S9C, D), these observations also strengthen the requirement for differential motility and differential cell-ECM sensitivity for cell sorting to occur. In accordance with this, cell sorting did not occur spheroids containing 4T1-mScarlet and 4T1 cells (Fig. S9E, F).

\section{Mixtures of cells with invadopodia and without invadopodia perform cooperative metastasis}

Dissemination and metastasis require transendothelial migration of cancer cells, i.e. intravasation and extravasation, which were previously shown to be invadopodia-dependent $(13,38)$. While interstitial ECM in the primary tumor and surrounding tissues may be permanently remodeled by microtracks formation (39), transendothelial migration involves brief, transient opening of perivascular ECM (40). It is not known whether invasive and non-invasive cells may also cooperate during metastasis. To investigate whether cells with invadopodia could enable metastasis of cells without invadopodia, we orthotopically injected individual metastatic or non-metastatic cell lines (4T1-CTL and 4T1-KD, as well as 4T1 and 67NR), and cell mixtures. After 14-20 days, we performed the clonogenic assay on digested lung tissues. To determine which cell type is growing colonies, we leveraged the difference in drug sensitivity. Namely, 4T1-KD cells are resistant to puromycin and geneticin, while 4T1-CTL cells are resistant to puromycin only; in addition, 4T1 cells are resistant to 6-thioguanine, while 67NR are resistant to geneticin. We confirmed that 4T1-KD and 67NR cells were not capable of lung metastasis, in contrast to 4T1-CTL and 4T1 cells (Fig. 5J). Importantly, when 4T1-KD cells were co-injected with 4T1 or 4T1-CTL cells they were 
capable of lung metastasis (Fig. 5K), suggesting that cooperative metastasis has occurred. Surprisingly, when $4 \mathrm{~T} 1$ and $67 \mathrm{NR}$ cells were co-injected, 67NR cells did not metastasize (Fig. 5K), suggesting that cooperative metastasis may be dependent on E-cadherin expression and/or presence of cell-cell junctions.

\section{Discussion}

In this study, we show that invasive cells can sort from and lead non-invasive cells during cooperative invasion and metastasis. By examining cell movements in spheroids, we demonstrate that differential persistence and ECM sensitivity drive cell sorting between clones. Through invadopodia removal, by silencing Tks5, we demonstrate that cells with invadopodia lead cells without invadopodia in cooperative invasion and enable their metastasis.

We confirm previously reported observations that the invasive 4T1 cells are in a hybrid epithelial/mesenchymal state, while the non-invasive 67NR cells are mesenchymal (27-29). It was previously shown that the expression of Twist, which is necessary for invadopodia (8), is present in 4T1 cells but not in 67NR cells (41). Hence, Twist-mediated EMT could be responsible for the differences in invasion skills between $4 T 1$ and $67 N R$ cells (41). Since $4 T 1$ cells but not $67 N R$ cells assemble functional invadopodia, it seems that specific invadopodia-granting EMT trajectory, rather than EMT completion is required for invadopodia emergence. Our results are in line with the recent evidence that epithelial cells (3, 42) and hybrid epithelial/mesenchymal cells can metastasize, sometimes more efficiently than mesenchymal cells $(43,44)$.

Despite expressing the core invadopodia components cortactin and Tks5, 67NR cells fail to degrade the matrix. However, 67NR cells lack active MMP-2 and MMP-9, likely due to MMP-14 (also known as MT1MMP) not being functional and/or not being delivered to the plasma membrane of these cells $(45,46)$.

For the first time here, we uncover the role of differential persistence and cell-ECM sensitivity in establishment of cell sorting in cancer. Classical studies on cell sorting during development showed that cell sorting relies on differential strength of cell-cell adhesions (33), or on differences in contractility (47). In contradiction to these views, we show that 4T1 and 67NR cells did not sort on a 2D gelatin layer or when placed in a 3D agarose matrix. The importance of differential motility in sorting was previously suggested during tissue patterning (48), and in engineered breast tubules, where it was shown that more directional epithelial cells accumulate at the tissue edge (49). During self-organization of mammary ducts, binary cell-ECM interactions (on or off) were reported to regulate cell sorting (36). Similar to our observations, Pawlizak et al. recently demonstrated that sorting of breast cell lines expressing $\mathrm{E}-, \mathrm{N}-$ or $\mathrm{P}-$ cadherin could not be explained by the differential adhesion hypothesis (50). Interestingly, the authors proposed that motility could be responsible for cell sorting.

We find that sorting precedes cooperative invasion, resulting in invasive cells accumulating at the spheroid-ECM interface and leading the invasion of multicellular strands. This emphasizes the importance of studying the mechanisms regulating the spatial organization of leader and follower cells. 
The accumulation of leader cells at the spheroid-matrix interface, rather than follower cells, may enhance the speed and efficiency of their cooperative invasion. Supporting these views, a recent study demonstrated that cell sorting precedes the basal extrusion of mammary epithelial cells (51).

Since the discovery of cooperative invasion, significant work has been carried out to uncover the mechanisms by which heterogeneous breast cancer cell populations interact and mobilize collectively. Our work suggests that invadopodia activity is a determinant of the leader cell phenotype. We previously showed that the G1 phase of the cell cycle is also a determinant of the leader cell identity (22). Consistent with this present work, we had also proved that invadopodia are enriched in the G1 phase of the cell cycle (22). A different study revealed that cells leading invasion strands possess higher intracellular energy compared to follower cells (17). Altogether, it seems that the cell cycle, intracellular energy and invadopodia function are determinants of the leader cell identity. However, the interplay between the cell cycle, intracellular energy and invadopodia function has yet to be investigated in the context of the emergence of leaders and followers within a cell population.

In summary, our study suggests that cooperativity between cancer clones may be an efficient mechanism for collective metastasis. Specifically, we demonstrate that invadopodia enable cooperative metastasis and allow non-invasive cells to metastasize. Our findings on cooperative metastasis of 4T1-KD/4T1 mixture are in alignment with the previous demonstration of dissemination of epithelial cell clusters (3). In contrast, we found that 67NR cells, which do not form heterotypic interactions with 4T1 cells, were not able to perform cooperative metastasis. To our knowledge, our work constitutes the first evidence for cooperative metastasis, which may be more detrimental than metastasis of single cells. We propose that targeting invadopodia could be a potent strategy to inhibit metastasis of both individually $(13,14)$, as well as collectively invading cells.

\section{Materials And Methods}

\section{Ethics statement}

All experiments on mice (Mus musculus) were conducted in accordance with the $\mathrm{NIH}$ regulations and approved by the Temple University IACUC protocol number 4766 .

\section{Fabrication of the spheroid imaging devices (SIDs)}

Spheroid imaging devices (SIDs) were fabricated as previously described (34). Briefly, SIDs were made by binding poly-dimethyl-siloxane (PDMS) disks to the glass-bottom dishes (MatTek Corporation). Each PDMS disk measures $17.5 \mathrm{~mm}$ in diameter and contains three wells, $5.5 \mathrm{~mm}$ in diameter, suited for individual spheroids.

\section{Gelatin coating of 6-well plates for cell culture}

6-well plates were coated with gelatin as previously described (52). Briefly, each well was coated with a $2.5 \%$ gelatin solution for $10 \mathrm{~min}$ followed by treatment with $0.5 \%$ glutaraldehyde (Sigma-Aldrich) for 10 
min, on ice, and an extra 30 min at room temperature. Plates were sterilized by $70 \%$ ethanol and then 50 $\mathrm{U} / \mathrm{mL}$ penicillin - $50 \mu \mathrm{g} / \mathrm{mL}$ streptomycin treatment.

\section{Cell culture}

The isogenic murine breast cancer cell lines 4T1 and 67NR were gifts from Dr. Fred R. Miller at the Karmanos Cancer Center and Dr. Jin Yang from UCSD. The human breast cancer cell line MDA-MB-231 (HTB-26) was obtained from the American Type Culture Collection. The MDA-MB-231-Dendra2-Tks5 KD cell line was described previously (13). All cells were cultured in Dulbecco's modified eagle medium [4.5 g/L D-glucose, L-glutamine] (DMEM, Gibco), supplemented with 10\% fetal bovine serum (FBS, Atlanta Biologicals) and $50 \mathrm{U} / \mathrm{mL}$ penicillin - $50 \mu \mathrm{g} / \mathrm{mL}$ streptomycin (Gibco). Cell cultures were maintained at 37 ${ }^{\circ} \mathrm{C}$ and $5 \% \mathrm{CO}_{2}$ for a maximum of 60 days.

\section{Plasmid transfections}

The 4T1-mScarlet and 67NR-GFP cell lines were generated by transfection using a 1:4 ratio of plasmid DNA:FugeneHD reagent (Promega), according to the manufacturer's instructions, followed by selection with $500 \mu \mathrm{g} / \mathrm{mL}$ geneticin (Fisher BioReagents) and $3 \mu \mathrm{g} / \mathrm{mL}$ puromycin (MP Biomedicals), respectively. pmScarlet-H-C1 was a gift from Dorus Gadella (Addgene plasmid \# 85043). pEGFP-puro was a gift from Michael McVoy (Addgene plasmid \# 45561). The MDA-MB-231-mScarlet cell line was generated by electroporation (Lonza) of the pmScarlet-C1 plasmid, according to the manufacturer's instructions, and selection with $500 \mu \mathrm{g} / \mathrm{ml}$ geneticin. After two weeks of drug selection, cells were sorted (BD FACSAriall $\mu$, BD Biosciences), collecting the subpopulations expressing high levels of mScarlet or GFP.

\section{Lentivirus transduction}

The Tks5 knockdown cell line 4T1-mScarlet-mTks5 KD and the knockdown control cell lines 4T1-shCTL and MDA-MB-231-mScarlet-shCTL were generated by transduction of the 4T1-mScarlet, 4T1 and MDAMB-231-mScarlet cell lines, respectively, with lentiviral particles (3 viral particles/cell) containing shRNA targeting mTks5 (KD) or non-targeting shRNA (CTL) in the pLKO.1-puro vector (MISSION library, SigmaAldrich), and selection with $2 \mu \mathrm{g} / \mathrm{mL}$ puromycin 3 days after infection.

\section{D proliferation assay and analysis}

Crystal violet staining was used to assess the effect of mitomycin $\mathrm{C}$ on the proliferation of the 4T1 cell line. Briefly, 4T1 cells were seeded in a 6-well plate and the next day, the culture medium was replaced with culture medium containing $0.5 \mu \mathrm{g} / \mathrm{mL}$ Mitomycin C (resuspended in DMSO, Cayman Chemical). After 2 days, cells were washed with cold PBS, fixed with ice-cold $100 \%$ methanol for 10 min and stained with $0.5 \%$ crystal violet solution in $25 \%$ methanol for 10 min at room temperature. Excess dye was removed by several washes with tap water and the plate was air dried overnight at room temperature. The dye was solubilized using $100 \%$ methanol for 20 min and the optical density was read on a plate reader 
at $570 \mathrm{~nm}$. The optical density at $570 \mathrm{~nm}$ for mitomycin C-treated cells was reported to the optical density at $570 \mathrm{~nm}$ for DMSO-treated cells.

\section{D gelatin degradation assay and immunofluorescence labeling}

Gelatin was fluorescently labeled with Alexa-405-NHS ester and $35 \mathrm{~mm}$ glass bottom dishes (MatTek Corporation) were coated with Alexa-405-gelatin as previously described (31). 400,000 (4T1/67NR) or 300,000 (MDA-MB-231) cells were plated per dish and cells were fixed $18 \mathrm{~h}$ later with $4 \%$ paraformaldehyde (Alfa Aesar) for $10 \mathrm{~min}$, permeabilized with $0.1 \%$ Triton X-100 (Calbiochem) for 5 min, blocked with 1\% FBS/1\% BSA (Sigma-Aldrich) in PBS (Gibco) for $3 \mathrm{~h}$, incubated with anti Tks5 antibody (Millipore, MABT336) for $2 \mathrm{~h}$, then with secondary antibody and Alexa Fluor 633 Phalloidin (Invitrogen) for $1 \mathrm{~h}$.

Samples were imaged on a laser scanning confocal microscope (FV1200, Olympus) using a 60X objective (UPLSAPO60XS, 1.35 NA, Olympus). Stacks were collected at $1 \mu \mathrm{m}$ z-step. To quantify matrix degradation, images were processed using a custom macro in Fiji. Briefly, slices from the stack were zprojected using the Max Intensity method, followed by thresholding of the signal in the gelatin channel, using the Automatic Threshold algorithm, and measuring the area of degradation spots using the Particle Analysis tool. To account for the differences in the cell density across fields of view, the total area of degradation in a field of view was divided by the total number of cell present in this field of view. Cells were counted using the F-actin staining.

\section{Scratch assay}

6-well plates were coated with $50 \mu \mathrm{g} / \mathrm{ml}$ poly-L-lysine (Sigma-Aldrich) for $20 \mathrm{~min}$ and air-dried. Cells were plated and cultured to confluency before a $10 \mu$ pipet tip was used to create a cross-shaped wound across the monolayer. Samples were imaged on a widefield microscope (IX-81, Olympus) equipped with an LED lamp (Excelitas Technologies), an Orca 16-bit charge-coupled device camera (Hamamatsu), an automated z-drift compensation IX3-ZDC (Olympus), an automated stage (Prior Scientific), an environmental chamber (Live Cell Instrument) and using a 10X objective (MPlanFL N 10X, 0.3 NA, Olympus). Cell motility was recorded at 10 min intervals over 48 hours. Manual cell tracking was performed using the TrackMate plugin through Fiji (53). The track number, the spot coordinates and the frame number were exported. Computation of the velocity and persistence were done using a custom made Matlab code.

\section{Generation of gelatin islands and 2D cell-ECM adhesive competition assay}

To generate gelatin islands, we utilized the PDMS inserts (see Fabrication of the spheroid imaging devices (SIDs)). Briefly, $35 \mathrm{~mm}$ glass bottom dishes (MatTek Corporation) were coated with $50 \mu \mathrm{g} / \mathrm{ml}$ poly-L-lysine (Sigma-Aldrich) for 20 min and air-dried. Then, PDMS rings were gently placed on top of the glass and sealed by gently pressing down. Next, each $5.5 \mathrm{~mm}$-diameter hole was coated with fluorescently labeled gelatin as previously described (31) (see 2D gelatin degradation assay and 
fluorescence labeling). 4T1-mScarlet and 67NR-GFP cells were plated in each hole at a 1 to 1 ratio. Cells were allowed to adhere for $1 \mathrm{~h}$, after which the PDMS inserts were gently peeled off the glass and medium was added to the dishes. Finally, cells were fixed $24 \mathrm{~h}$ later with $4 \%$ paraformaldehyde (Alfa Aesar) for $10 \mathrm{~min}$.

Samples were imaged on a widefield microscope (Eclipse Ti2-E, Nikon) equipped with a pco.panda sCMOS camera (PCO) and using a 10X objective (CFI Plan Fluor 10X, 0.3 NA, Nikon). Tiles (6x6) were acquired to visualize the entire gelatin island surface. Live cells were imaged every 10 min and using an environmental chamber (Tokai Hit). The movie was annotated using the DrawArrowInMovie Fiji plugin (54). To quantify the 2D cell-ECM adhesive competition assay, we counted the number of 4T1 and 67NR cells that migrated off the gelatin islands. To quantify cell sorting in 2D, we only utilized regions coated with gelatin and we counted the number of homotypic neighbors.

\section{Measurements of the cell-ECM contact angle}

Measurement of the contact angle was performed as previously described (36). Briefly, $35 \mathrm{~mm}$ glass bottom dishes (MatTek Corporation) were coated with fluorescently labeled gelatin as previously described (31) or with $50 \mu \mathrm{g} / \mathrm{ml}$ poly-L-lysine (Sigma-Aldrich) for $20 \mathrm{~min}$ and let to air dry. Cells were left to adhere for $5 \mathrm{~h}$ before imaging. Solitary cells, which had no physical interaction with nearby cells, were imaged on a laser scanning confocal microscope (FV1200, Olympus) using a 60X objective (UPLSAP060XS, 1.35 NA, Olympus) equipped with an environmental chamber (In Vivo Scientific), with a $1 \mu \mathrm{m}$ z-step. Orthogonal Views was used to measure the angle between the ECM and the main body of the cell: the contact angle.

\section{D spheroid invasion assays}

For 4T1 and 67NR cell lines, 3D spheroids were generated by the hanging drop method. 3,000 cells per 40 $\mu \mathrm{l}$ drop containing $4.8 \mathrm{mg} / \mathrm{mL}$ methylcellulose (Sigma-Aldrich), $20 \mu \mathrm{g} / \mathrm{mL}$ Nutragen (Advanced Biomatrix), were placed on the lid of tissue culture dishes. The lids were carefully turned and placed on the bottom reservoir of the dishes filled with PBS to prevent evaporation. Alternatively, for MDA-MB-231 cell lines, 3D spheroids were generated in a 96-well V-bottom dish coated with $0.5 \%$ poly(2-hydroxyethyl methacrylate) (Sigma-Aldrich) in ethanol. Then, 5,000 cells in $50 \mu \mathrm{l}$ of medium were distributed to each well and the plate was centrifuged for $20 \mathrm{~min}$ at $1,000 \times \mathrm{g}$ and at $4^{\circ} \mathrm{C}$. Finally, $50 \mu \mathrm{l}$ of Matrigel (Corning) was added to each well at a final concentration of $2.5 \%$. The spheroids were formed over 3 days at $37^{\circ} \mathrm{C}$ and $5 \% \mathrm{CO}_{2}$, embedded in $30 \mu \mathrm{l}$ of $5 \mathrm{mg} / \mathrm{mL}$ rat tail Collagen I (Corning, alternate gelation protocol) and placed into the SIDs. Collagen I was polymerized at $37^{\circ} \mathrm{C}$ for $30 \mathrm{~min}$ and then culture medium was added to the dishes. For drug treatments, cell culture medium containing $25 \mu \mathrm{M} \mathrm{GM6001} \mathrm{(resuspended} \mathrm{in}$ DMSO, Cayman Chemical), $10 \mu \mathrm{M}$ Y-27632 (resuspended in DMSO, Cayman Chemical) or $0.1 \%$ DMSO control was used.

For the experiments where 4T1-mScarlet cells surround the 67NR-GFP spheroids, 4T1-mScarlet cells were added to the collagen mix containing the 67 NR-GFP spheroids at $10^{\wedge} 6$ cells $/ \mathrm{mL}$ before polymerization of 
the collagen.

For the experiments where conditioned medium was used, 4T1-mScarlet cells were seeded onto a gelatincoated 6-well plate, at $2 \times 10^{6}$ cells/well. $2 \mathrm{~mL}$ of complete DMEM were used per well and the embedded 67NR-GFP spheroids were cultured, from day 0 , using the conditioned medium from the 4T1-mScarlet cells plated onto gelatin. Every two days, the conditioned medium was replaced.

Collagen was labeled as previously described (55), using $2 \mu \mathrm{g} / \mathrm{ml}$ of 405-Alexa-NHS ester (Biotium).

\section{Immunolabeling, imaging and analysis of fixed spheroids}

Immunofluorescence labeling was performed as previously described (34). Briefly, the embedded spheroids were simultaneously fixed and permeabilized in 4\% paraformaldehyde and $0.5 \%$ Triton X-100 in PBS for 5 min, further fixed in 4\% paraformaldehyde in PBS for 20 min and blocked in 1\% FBS/1\% BSA in PBS at $4{ }^{\circ} \mathrm{C}$ for $24 \mathrm{~h}$ on a shaker. The embedded spheroids were then incubated with the anti-collagen I $3 / 4$ (immunoGlobe, 0207-050), anti E-cadherin (Invitrogen, 13-1900), anti N-cadherin (BD Transduction Laboratories, 610920) and anti Tks5 (Millipore, MABT336) overnight at $4{ }^{\circ} \mathrm{C}$ and with secondary antibodies and Alexa Fluor 633 Phalloidin (Invitrogen) for $1 \mathrm{~h}$ at room temperature on a shaker.

Spheroids were imaged using a laser scanning confocal microscope (FV1200, Olympus) with a 10X (UPLXAP010X, 0.4 NA, Olympus) or a 30X objective (UPLSAPO30XSIR, 1.05 NA, Olympus), using a 3-5 $\mu \mathrm{m} z$-step.To quantify the total spheroid area, spheroids were labeled with DAPI. The images were processed using a custom macro in Fiji. Briefly, slices were z-projected using the Max Intensity method and the nuclei were selected using the Automatic Threshold algorithm from Fiji. Then, the Particle Analysis tool was used to measure the total area of nuclei.

To quantify the E-cadherin signal, the slices of interest were z-projected using the Max Intensity method and strands or single cells were identified. For the relative junction/cytosol ratio: a $10 \mu \mathrm{m}$-long line was drawn across and centered at the junction between two cells within a strand. The gray value along the line was measured for the E-cadherin and F-actin channel using the Plot Profile tool. The gray value at 0 and $5 \mu \mathrm{m}$ were defined as the "cytosol" and "junction" signal, respectively.

To quantify cell sorting, images were processed using a custom macro in Fiji. Since maximum projection of the z-slices introduces artefacts in the positions of cells, regarding the spheroid edge $v s$. core compartments, we utilized the median slice of the z-stack only. Briefly, the median slice was extracted from the z-stack and the spheroid core was selected in the brightfield channel using the Automatic Threshold algorithm from Fiji. Then, using the Fit Ellipse and Centroid options in Measurements, the coordinates of the spheroid core center and the major axis of the spheroid core were extracted. Finally, the Multi-point tool was used to record the coordinates of GFP+ and mScarlet+ cells. Cell sorting was quantified as the distance from the spheroid center to the cell (d in Fig. 2C) over the semi major axis of the spheroid (a in Fig. 2C). We defined this ratio as the "Distance Index" (DI). Alternatively, for mixed spheroids that contained non-labeled cells, like 4T1-shCTL (Fig. 5) or 4T1 cells (Fig. S7), DAPI staining 
was used to measure a cell's coordinate. For a given tracked cell, we defined the DDI as the DI for its final position minus the DI for its initial position.

\section{Live imaging of spheroids and image processing}

Live imaging of spheroids was performed either longitudinally (daily), to analyze cell sorting, or via timelapse, to analyze cell motility. Confocal microscope (FV1200, Olympus) using a 10X objective (UPLXAP010X, 0.4 NA, Olympus) equipped with an environmental chamber (Live Cell instrument) was used. Cell motility was recorded at 10- or 20-min intervals over 44 hours, with a $15 \mu \mathrm{m}$ z-step. Only slices where both the cells from the core and the edge compartments were visible, were used for tracking. Cell tracking was performed using the TrackMate plugin through Fiji (53). Spot detection was done using the LoG detector with median filtering and subpixel localization. Then, the linear motion LAP tracker was used to link spots. Tracks were filtered based on the number of spots in the track with a $\geq 5$ spots/track cutoff. Tracks were visually validated and corrected if needed, using the TrackScheme tool. Finally, gaps in tracks were closed by introducing new spots. The new spots position was calculated using linear interpolation. For each track, the distance from the original spot to the spheroid-collagen I interface was measured and if this distance was $\leq 30 \mu \mathrm{m}$, the track was classified as edge, otherwise the track was classified as core. The track number, the spot coordinates and the frame number were exported. Computation of the distance index was done using a custom made Matlab code (available upon request).

\section{Mean-squared displacement analysis of cells}

Coordinates of individual cell trajectories were obtained by particle tracking with the origin of each spheroid at $(0,0)$, and were transformed from the Cartesian $\{x, y\}$ to $\{r, f\}$ polar coordinate system. Mean squared displacements (MSDs) were then calculated in radial and angular directions, averaged over different spheroids and experimental repeats for different conditions. The MSD data were then fit to a power law, in both directions, namely 


$$
\left\langle\left(r-r_{0}\right)^{2}\right\rangle=\Gamma_{\mathrm{r}} \mathrm{t}^{\alpha_{\mathrm{r}}},
$$

and

$$
\left\langle\left(\phi-\phi_{0}\right)^{2}\right\rangle=\Gamma_{\phi} \mathrm{t}^{\alpha_{\phi}},
$$

where $\left(r_{0}, \phi_{0}\right)$ correspond to the origin of each trajectory in the polar coordinate system, $\Gamma_{r, \phi}$ correspond to the amplitudes of the power laws, and $\alpha_{r, \phi}$ are the exponents, in radial and angular directions, respectively. MSD data were fit to these power laws using Levenberg-Marquart algorithm, with the range of fits in the interval $[0,3 \mathrm{~h}]$. Note that the motility of cells is sub-diffusive for $\alpha<1$, diffusive for $\alpha=1$, and super-diffusive for $\alpha>1$.

In the radial direction, since the motion was found to be super-diffusive, we also calculated the time-dependent effective diffusion coefficient given by (56)

resulting in

$$
D_{e f f}(t)=(\Gamma / 2) t^{\alpha-1},
$$

$$
\left\langle\left(r-r_{0}\right)^{2}\right\rangle=2 \mathrm{D}_{\text {eff }}(\mathrm{t}) t .
$$

It is important to note that, for motion that is not diffusive, time-dependent $D_{\text {eff }}(t)$ is the only way to estimate a diffusion coefficient with the correct units, and compare different data sets in a consistent way, as long as the same time points are chosen for comparison.

\section{Western blot assay}

Cells cultured to $80 \%$ confluency were harvested in ice-cold RIPA lysis buffer (Teknova), supplemented with protease inhibitors (complete cocktail, Roche) and phosphatase inhibitors (Halt cocktail, SigmaAldrich). SDS-PAGE was performed with $20 \mu \mathrm{g}$ protein per sample, transferred to a polyvinylidene difluoride membrane (Immobilon), blocked with 5\% BSA/TBST for $3 \mathrm{~h}$ at room temperature and incubated with anti-b-actin (Santa Cruz Biotechnology, sc-47778), anti-cortactin (Abcam, ab33333), anti-E-cadherin (BD Transduction Laboratories, 610181), anti-FAK (Santa Cruz Biotechnology, sc-271126), anti-N-cadherin (BD Transduction Laboratories, 610920) and anti-Tks5 (Millipore, MABT336) antibodies diluted in 5\% BSA/TBST overnight at $4{ }^{\circ} \mathrm{C}$. The membranes were then incubated with HRP conjugated anti-mouse or anti-rabbit IgG (Cell Signaling Technologies) antibodies diluted in 5\% non-fat milk/TBST for $1 \mathrm{~h}$ at room temperature and proteins were visualized using chemiluminescence detection reagents (WesternBright, Advansta) and blot scanner (C-DiGit, LI-COR).

\section{Lung clonogenic assay}

The lung clonogenic assay was performed as previously described (57). Briefly, 200,000 cells were suspended in $100 \mu \mathrm{l}$ of $20 \%$ collagen I in PBS and injected orthotopically into the mammary fat pad of 7week old female Balb/cJ mice. For inoculation with mixtures of $4 \mathrm{~T} 1$ cells, the cell ratio was of 1:1. For inoculation with 4T1:67NR cell mixtures, the cell ratio was of 1:300. After 14-20 days, when the tumor diameter reached 8-12 $\mathrm{mm}$, the animals were sacrificed and the lungs harvested. The lungs were minced, digested in a collagenase type IV/elastase cocktail (Worthington Biochemical) and filtered through a 70 $\mu \mathrm{m}$ mesh. Then, the cell suspension from each lung was split into 2 tissue culture plates and incubated with a combination of 6-thioguanine (Cayman Chemicals) and puromycin, for growth of 4T1-shControl or 4T1-mScarlet-mTks5 KD cells; or with a combination of 6-thioguanine, puromycin and geneticin for 
growth of $4 \mathrm{~T} 1$-mScarlet-mTks5 KD cells only. After 14 days at $37^{\circ} \mathrm{C}$ and $5 \% \mathrm{CO}_{2}$, the colonies were stained using $0.03 \%(\mathrm{w} / \mathrm{v})$ methylene blue (Sigma-Aldrich) and counted.

\section{Statistical analyses}

RStudio software was used to perform all statistical analyses. The distribution of each data set was analyzed, and the Shapiro-Wilk test was performed to test for normality. For normally distributed data sets, $F$ test was performed to compare the variances of two data sets. Based on the results from the $F$ test, a Welch two-sample t-test or a two-sample t-test was done to compare the means of the two data sets. For non-normally distributed data sets a Wilcoxon rank sum test was performed to compare the two data sets. Unless stated otherwise, all tests were performed using unpaired and two-sided criteria. Statistical significance was defined as ${ }^{*} p<0.05,{ }^{* *} p<0.01$, and ${ }^{* *} p<0.001$. Additional information on the metrics and statistics can be found in the Table S1.

\section{Declarations}

\section{Acknowledgments}

We would like to thank members of Temple Bioengineering and Fox Chase Cancer Center Biology for valuable discussions. We thank David Ambrose at the flow cytometry core (Lewis Katz School of Medicine) for his assistance with cell sorting.

\section{Funding:}

American Cancer Society Research Scholar Grant 134415-RSG-20-034-01-CSM (BG) Conquer Cancer Now/Young Investigator Award (BG)

National Institutes of Health grant R00 CA172360 (BG)

National Institutes of Health grant R01 CA230777 (BG)

National Institutes of Health grant R01 GM121679 (ET)

\section{Author contributions:}

Conceptualization: LP, BG

Data acquisition: LP, BB, BG

Analysis: LP, ET

Supervision: BG

Writing: LP, BB, ET, BG 
Competing interests: Authors declare that they have no competing interests.

Data and materials availability: All data, code, and materials that are not commercially available will be available upon request.

\section{References}

1. Howlader N, Noone A, Krapcho M, Miller D, Brest A, Yu M, Ruhl J, Tatalovich Z, Mariotto A, Lewis D, Chen H, Feuer E, Cronin K. SEER Cancer Statistics Review, 1975-2017, based on November 2019 SEER data submission [Internet]. National Cancer Institute. Bethesda, MD.

2. Vanharanta S, Massagué J. Origins of Metastatic Traits. Cancer Cell. (2013) 24:410-21.

3. Cheung KJ, Padmanaban V, Silvestri V, Schipper K, Cohen JD, Fairchild AN, Gorin MA, Verdone JE, Pienta KJ, Bader JS, Ewald AJ. Polyclonal breast cancer metastases arise from collective dissemination of keratin 14-expressing tumor cell clusters. Proc Natl Acad Sci. (2016) 113:E854-63.

4. Aceto N, Bardia A, Miyamoto DT, Donaldson MC, Wittner BS, Spencer JA, Yu M, Pely A, Engstrom A, Zhu H, Brannigan BW, Kapur R, Stott SL, Shioda T, Ramaswamy S, Ting DT, Lin CP, Toner M, Haber DA, Maheswaran S. Circulating Tumor Cell Clusters Are Oligoclonal Precursors of Breast Cancer Metastasis. Cell. (2014) 158:1110-22.

5. Tabassum DP, Polyak K. Tumorigenesis: it takes a village. Nat Rev Cancer. (2015) 15:473-83.

6. Yeung KT, Yang J. Epithelial-mesenchymal transition in tumor metastasis. Mol Oncol. (2017) 11:28-39.

7. Pignatelli J, Tumbarello DA, Schmidt RP, Turner CE. Hic-5 promotes invadopodia formation and invasion during TGF- $\beta$-induced epithelial-mesenchymal transition. J Cell Biol. (2012) 197:421-37.

8. Eckert MA, Lwin TM, Chang AT, Kim J, Danis E, Ohno-Machado L, Yang J. Twist1-Induced Invadopodia Formation Promotes Tumor Metastasis. Cancer Cell. (2011) 19:372-86.

9. Eckert MA, Santiago-Medina M, Lwin TM, Kim J, Courtneidge SA, Yang J. ADAM12 induction by Twist1 promotes tumor invasion and metastasis via regulation of invadopodia and focal adhesions. $J$ Cell Sci. (2017) 130:2036-48.

10. Karamanou K, Franchi M, Vynios D, Brézillon S. Epithelial-to-mesenchymal transition and invadopodia markers in breast cancer: Lumican a key regulator. Semin Cancer Biol. (2020) 62:125-33.

11. Murphy DA, Courtneidge SA. The "ins" and "outs" of podosomes and invadopodia: characteristics, formation and function. Nat Rev Mol Cell Biol. (2011) 12:413-26. 
12. Eddy RJ, Weidmann MD, Sharma VP, Condeelis JS. Tumor Cell Invadopodia: Invasive Protrusions that Orchestrate Metastasis. Trends Cell Biol. (2017) 27:595-607.

13. Gligorijevic B, Bergman A, Condeelis J. Multiparametric classification links tumor microenvironments with tumor cell phenotype. PLoS Biol. (2014) 12:e1001995.

14. Gligorijevic B, Wyckoff J, Yamaguchi H, Wang Y, Roussos ET, Condeelis J. N-WASP-mediated invadopodium formation is involved in intravasation and lung metastasis of mammary tumors. $J$ Cell Sci. (2012) 125:724-34.

15. Ye X, Weinberg RA. Epithelial-Mesenchymal Plasticity: A Central Regulator of Cancer Progression. Trends Cell Biol. (2015) 25:675-86.

16. Cheung KJ, Gabrielson E, Werb Z, Ewald AJ. Collective invasion in breast cancer requires a conserved basal epithelial program. Cell. (2013) 155:1639-51.

17. Zhang J, Goliwas KF, Wang W, Taufalele P V., Bordeleau F, Reinhart-King CA. Energetic regulation of coordinated leader-follower dynamics during collective invasion of breast cancer cells. Proc Natl Acad Sci. (2019) 116:7867-72.

18. Wolf K, Wu Yl, Liu Y, Geiger J, Tam E, Overall C, Stack MS, Friedl P. Multi-step pericellular proteolysis controls the transition from individual to collective cancer cell invasion. Nat Cell Biol. (2007) 9:893-904.

19. Carey SP, Starchenko A, McGregor AL, Reinhart-King CA. Leading malignant cells initiate collective epithelial cell invasion in a three-dimensional heterotypic tumor spheroid model. Clin Exp Metastasis. (2013) 30:615-30.

20. Westcott JM, Prechtl AM, Maine EA, Dang TT, Esparza MA, Sun H, Zhou Y, Xie Y, Pearson GW. An epigenetically distinct breast cancer cell subpopulation promotes collective invasion. J Clin Invest. (2015) 125:1927-43.

21. Chapman A, Fernandez del Ama L, Ferguson J, Kamarashev J, Wellbrock C, Hurlstone A. Heterogeneous Tumor Subpopulations Cooperate to Drive Invasion. Cell Rep. (2014) 8:688-95.

22. Bayarmagnai B, Perrin L, Esmaeili Pourfarhangi K, Graña X, Tüzel E, Gligorijevic B. Invadopodiamediated ECM degradation is enhanced in the G1 phase of the cell cycle. J Cell Sci. (2019) 132:jcs227116.

23. Miller BE, Miller FR, Wilburn DJ, Heppner GH. Analysis of tumour cell composition in tumours composed of paired mixtures of mammary tumour cell lines. Br J Cancer. (1987) 56:561-9.

24. Dexter DL, Kowalski HM, Blazar BA, Fligiel Z, Vogel R, Heppner GH. Heterogeneity of tumor cells from a single mouse mammary tumor. Cancer Res. (1978) 38:3174-81. 
25. Aslakson CJ, Miller FR. Selective events in the metastatic process defined by analysis of the sequential dissemination of subpopulations of a mouse mammary tumor. Cancer Res. (1992) 52:1399405.

26. Seki Y, Toba K, Fuse I, Sato N, Niwano H, Takahashi H, Tanabe N, Aizawa Y. In vitro effect of cyclosporin $\mathrm{A}$, mitomycin $\mathrm{C}$ and prednisolone on cell kinetics in cultured human umbilical vein endothelial cells. Thromb Res. (2005) 115:219-28.

27. Truong HH, Xiong J, Ghotra VPS, Nirmala E, Haazen L, Le Devedec SE, Balcio lu HE, He S, SnaarJagalska BE, Vreugdenhil E, Meerman JHN, van de Water B, Danen EHJ. 1 Integrin Inhibition Elicits a Prometastatic Switch Through the TGF -miR-200-ZEB Network in E-Cadherin-Positive Triple-Negative Breast Cancer. Sci Signal. (2014) 7:ra15-ra15.

28. Ilina O, Campanello L, Gritsenko PG, Vullings M, Wang C, Bult P, Losert W, Friedl P. Intravital microscopy of collective invasion plasticity in breast cancer. Dis Model Mech. (2018) 11:dmm034330.

29. Lou Y, Preobrazhenska O, Auf Dem Keller U, Sutcliffe M, Barclay L, McDonald PC, Roskelley C, Overall CM, Dedhar S. Epithelial-Mesenchymal Transition (EMT) is not sufficient for spontaneous murine breast cancer metastasis. Dev Dyn. (2008) 237:2755-68.

30. Konen J, Summerbell E, Dwivedi B, Galior K, Hou Y, Rusnak L, Chen A, Saltz J, Zhou W, Boise LH, Vertino P, Cooper L, Salaita K, Kowalski J, Marcus Al. Image-guided genomics of phenotypically heterogeneous populations reveals vascular signalling during symbiotic collective cancer invasion. Nat Commun. (2017) 8:15078.

31. Sharma VP, Entenberg D, Condeelis J. High-Resolution Live-Cell Imaging and Time-Lapse Microscopy of Invadopodium Dynamics and Tracking Analysis. In: Coutts AS, editor. Methods in molecular biology (Clifton, NJ). Totowa, NJ: Humana Press; p. 343-57. (Methods in Molecular Biology; vol. 1046).

32. Pourfarhangi KE, Bergman A, Gligorijevic B. ECM Cross-Linking Regulates Invadopodia Dynamics. Biophys J. (2018) 114:1455-66.

33. Steinberg MS. Reconstruction of Tissues by Dissociated Cells. Science (80-). (1963) 141:401-8.

34. Perrin L, Tucker T, Gligorijevic B. Time-Resolved Fluorescence Imaging and Analysis of Cancer Cell Invasion in the 3D Spheroid Model. J Vis Exp. (2021):1-14.

35. Smyrek I, Mathew B, Fischer SC, Lissek SM, Becker S, Stelzer EHK. E-cadherin, actin, microtubules and FAK dominate different spheroid formation phases and important elements of tissue integrity. Biol Open. (2019) 8:bio037051.

36. Cerchiari AE, Garbe JC, Jee NY, Todhunter ME, Broaders KE, Peehl DM, Desai TA, LaBarge MA, Thomsonf M, Gartner ZJ. A strategy for tissue self-organization that is robust to cellular heterogeneity 
and plasticity. Proc Natl Acad Sci U S A. (2015) 112:2287-92.

37. Labernadie A, Kato T, Brugués A, Serra-Picamal X, Derzsi S, Arwert E, Weston A, González-Tarragó V, Elosegui-Artola A, Albertazzi L, Alcaraz J, Roca-Cusachs P, Sahai E, Trepat X. A mechanically active heterotypic E-cadherin/ $\mathrm{N}$-cadherin adhesion enables fibroblasts to drive cancer cell invasion. Nat Cell Biol. (2017) 19:224-37.

38. Leong HS, Robertson AE, Stoletov K, Leith SJ, Chin CA, Chien AE, Hague MN, Ablack A, CarmineSimmen K, McPherson VA, Postenka C, Turley EA, Courtneidge SA, Chambers AF, Lewis JD. Invadopodia Are Required for Cancer Cell Extravasation and Are a Therapeutic Target for Metastasis. Cell Rep. (2014) 8:1558-70.

39. Ilina O, Gritsenko PG, Syga S, Lippoldt J, La Porta CAM, Chepizhko O, Grosser S, Vullings M, Bakker G-J, Starruß J, Bult P, Zapperi S, Käs JA, Deutsch A, Friedl P. Cell-cell adhesion and 3D matrix confinement determine jamming transitions in breast cancer invasion. Nat Cell Biol. (2020).

40. Harney AS, Arwert EN, Entenberg D, Wang Y, Guo P, Qian B-Z, Oktay MH, Pollard JW, Jones JG, Condeelis JS. Real-Time Imaging Reveals Local, Transient Vascular Permeability, and Tumor Cell Intravasation Stimulated by TIE2hi Macrophage-Derived VEGFA. Cancer Discov. (2015) 5:932-43.

41. Yang J, Mani SA, Donaher JL, Ramaswamy S, Itzykson RA, Come C, Savagner P, Gitelman I, Richardson A, Weinberg RA. Twist, a Master Regulator of Morphogenesis, Plays an Essential Role in Tumor Metastasis. Cell. (2004) 117:927-39.

42. Padmanaban V, Krol I, Suhail Y, Szczerba BM, Aceto N, Bader JS, Ewald AJ. E-cadherin is required for metastasis in multiple models of breast cancer. Nature. (2019) 573:439-44.

43. Aiello NM, Maddipati R, Norgard RJ, Balli D, Li J, Yuan S, Yamazoe T, Black T, Sahmoud A, Furth EE, Bar-Sagi D, Stanger BZ. EMT Subtype Influences Epithelial Plasticity and Mode of Cell Migration. Dev Cell. (2018) 45:681-695.e4.

44. Nieto MA, Huang RYYJ, Jackson RAA, Thiery JPP. Emt: 2016. Cell. (2016) 166:21-45.

45. Tester AM, Ruangpanit N, Anderson RL, Thompson EW. MMP-9 secretion and MMP-2 activation distinguish invasive and metastatic sublines of a mouse mammary carcinoma system showing epithelial-mesenchymal transition traits. Clin Exp Metastasis. (2000) 18:553-60.

46. Johnstone CN, Smith YE, Cao Y, Burrows AD, Cross RSN, Ling X, Redvers RP, Doherty JP, Eckhardt BL, Natoli AL, Restall CM, Lucas E, Pearson HB, Deb S, Britt KL, Rizzitelli A, Li J, Harmey JH, Pouliot N, Anderson RL. Functional and molecular characterisation of EO771.LMB tumours, a new C57BL/6-mousederived model of spontaneously metastatic mammary cancer. Dis Model Mech. (2015) 8:237-51.

47. Heisenberg C-P, Bellaïche Y. Forces in Tissue Morphogenesis and Patterning. Cell. (2013) 153:94862. 
48. Beatrici CP, Brunnet LG. Cell sorting based on motility differences. Phys Rev E. (2011) 84:031927.

49. Mori H, Gjorevski N, Inman JL, Bissell MJ, Nelson CM. Self-organization of engineered epithelial tubules by differential cellular motility. Proc Natl Acad Sci. (2009) 106:14890-5.

50. Pawlizak S, Fritsch AW, Grosser S, Ahrens D, Thalheim T, Riedel S, Kießling TR, Oswald L, Zink M, Manning ML, Käs JA. Testing the differential adhesion hypothesis across the epithelial-mesenchymal transition. New J Phys. (2015) 17:083049.

51. Villeneuve C, Lagoutte E, de Plater L, Mathieu S, Manneville J-B, Maître J-L, Chavrier P, Rossé C. aPKCi triggers basal extrusion of luminal mammary epithelial cells by tuning contractility and vinculin localization at cell junctions. Proc Natl Acad Sci. (2019) 116:24108-14.

52. Chen $\mathrm{W}-\mathrm{T}$, Yeh $\mathrm{Y}$, Nakahara $\mathrm{H}$. An in vitro cell invasion assay: Determination of cell surface proteolytic activity that degrades extracellular matrix. J Tissue Cult Methods. (1994) 16:177-81.

53. Tinevez J-Y, Perry N, Schindelin J, Hoopes GM, Reynolds GD, Laplantine E, Bednarek SY, Shorte SL, Eliceiri KW. TrackMate: An open and extensible platform for single-particle tracking. Methods. (2017) 115:80-90.

54. Daetwyler S, Modes CD, Fiolka R. Fiji plugin for annotating movies with custom arrows. Biol Open. (2020) 9:3-6.

55. Di Martino J, Henriet E, Ezzoukhry Z, Mondal C, Bravo-Cordero JJ, Moreau V, Saltel F. 2D and 3D Matrices to Study Linear Invadosome Formation and Activity. J Vis Exp. (2017):1-9.

56. Wu J, Berland KM. Propagators and Time-Dependent Diffusion Coefficients for Anomalous Diffusion. Biophys J. (2008) 95:2049-52.

57. Pulaski BA, Ostrand-Rosenberg S. Mouse 4T1 Breast Tumor Model. Curr Protoc Immunol. (2000) 39:1-16.

\section{Figures}



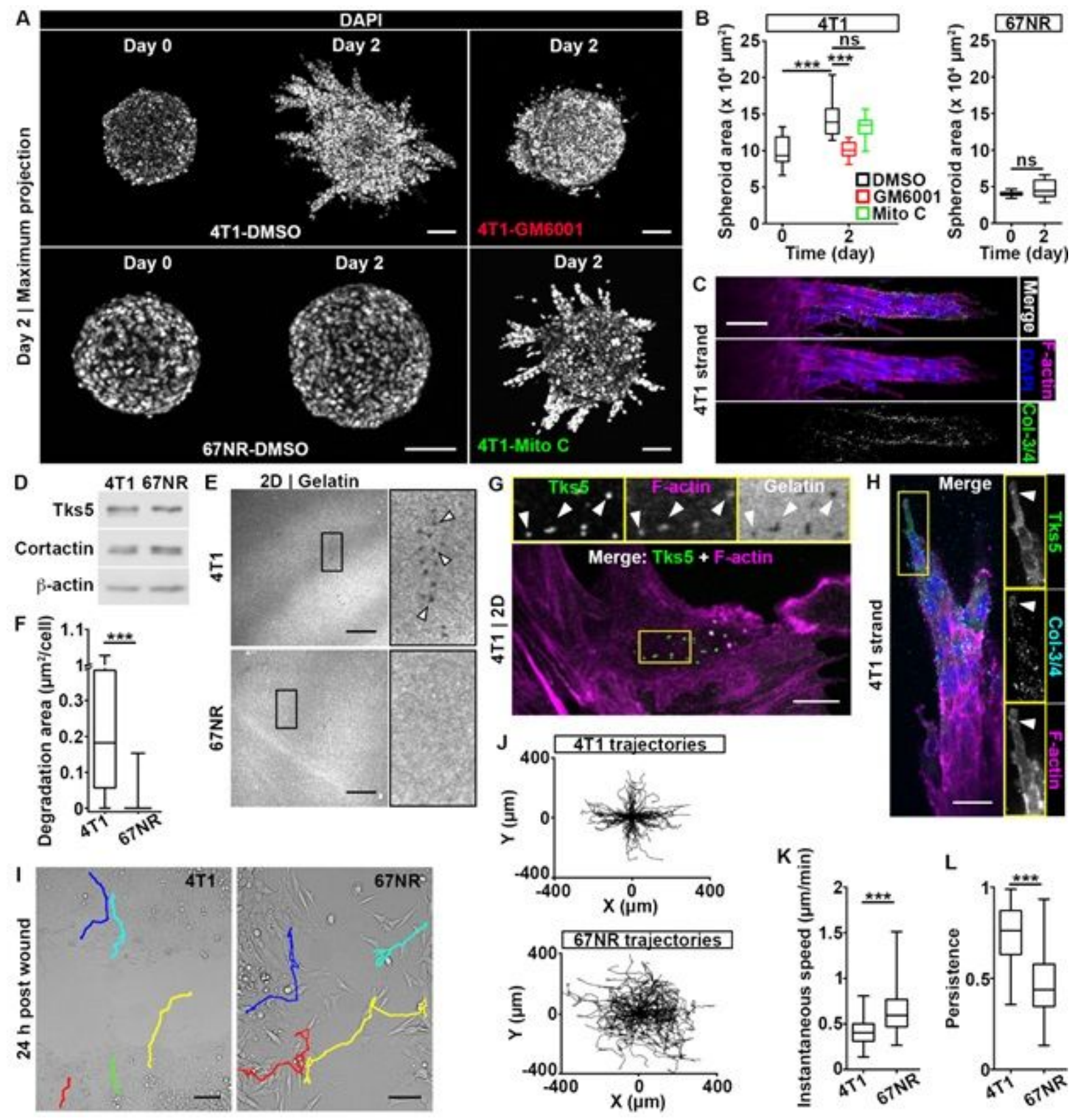

\section{Figure 1}

4T1 cells, but not 67NR cells, exhibit MMP-dependent spheroid invasion. (A) Representative micrographs (maximum projection) of 4T1 and 67NR spheroids cultured in a 3D collagen I matrix for 2 days. Nuclei were stained with DAPI. Spheroids were treated from day 0 with a pan-MMP inhibitor (GM6001, top right panel), a cell cycle inhibitor (mitomycin C, Mito C, bottom right panel) or DMSO control. Scale bar: 100 $\mu \mathrm{m}$. (B) Quantification of the spheroid area as a function of time for 4T1 and 67NR cells from (A). $P=0.000581,0.000123,0.125$ and 0.0571 were obtained by the two-sample t-test. (C) Representative micrographs of a $4 \mathrm{~T} 1$ strand 2 days after the spheroid was embedded in a 3D collagen I matrix. The spheroid was immunolabeled for MMP-mediated cleaved collagen I (Col-3/4, green). F-actin (phalloidin, magenta) and nuclei (DAPI, blue) were stained. Scale bar: $50 \mu \mathrm{m}$. (D) Western blot analysis of Tks5 and cortactin expression levels in $4 \mathrm{~T} 1$ and $67 \mathrm{NR}$ cells. $\beta$-actin is used as a loading control. (E) Representative 
micrographs of gelatin degradation for 4T1 (top panel) and 67NR (bottom panel) cells $18 \mathrm{~h}$ post plating. The insets show a $4 \mathrm{X}$ zoom-in of the boxed area and arrowheads indicate representative degradation holes. Scale bars: $20 \mu \mathrm{m}$. (F) Quantification of the degradation area per cell for 4T1 and 67NR cells from (E). $P<4.40 \times 10-16$ was obtained by the Wilcoxon rank sum test. (G) Representative micrographs of 4T1 cells cultured on fluorescent gelatin (gray), immunolabeled for Tks5 (green) and with labeled F-actin (phalloidin, magenta). The insets show a $2 \mathrm{X}$ zoom-in of the boxed area and arrowheads indicate representative functional invadopodia. Scale bar: $10 \mu \mathrm{m}$. (H) Representative micrographs of a 4T1 strand 2 days after the spheroid was embedded in a 3D collagen I matrix. The spheroid was immunolabeled for Tks5 (green) and MMP-mediated cleaved collagen I (Col-3/4, cyan). F-actin (phalloidin, magenta) and nuclei (DAPI, blue) were stained. The insets show a 1.25X zoom-in of the boxed area. Scale bar: $30 \mu \mathrm{m}$. (I) Representative snapshot micrographs of a 4T1 (left panel) and a 67NR (right panel) monolayer $24 \mathrm{~h}$ post wounding. Representative cell trajectories are shown. Scale bars: $100 \mu \mathrm{m}$. (J) Trajectories of 4T1 (top panel) and 67NR (bottom panel) cells from the wound assay shown as wind-rose plots. The trajectories are shifted to a common origin. (K) Quantification of the instantaneous speed of 4T1 and 67NR cells from $(J)$. $P=1.17 \times 10-13$ was obtained by the Wilcoxon rank sum test. ( $L$ ) Quantification of the persistence (net displacement/path length) of $4 \mathrm{~T} 1$ and 67 NR cells from $(\mathrm{J}) . \mathrm{P}<2.20 \times 10-16$ was obtained by the Wilcoxon rank sum test. All data are represented as boxplots with median (line), 25th/75th percentiles (boxes) and maximum/minimum (whiskers). Statistical significance was defined as ${ }^{\star} p<0.05,{ }^{\star \star} p<0.01$ and ${ }^{* \star} p<0.001$. Additional information on the metrics and statistics can be found in the Table S1. 

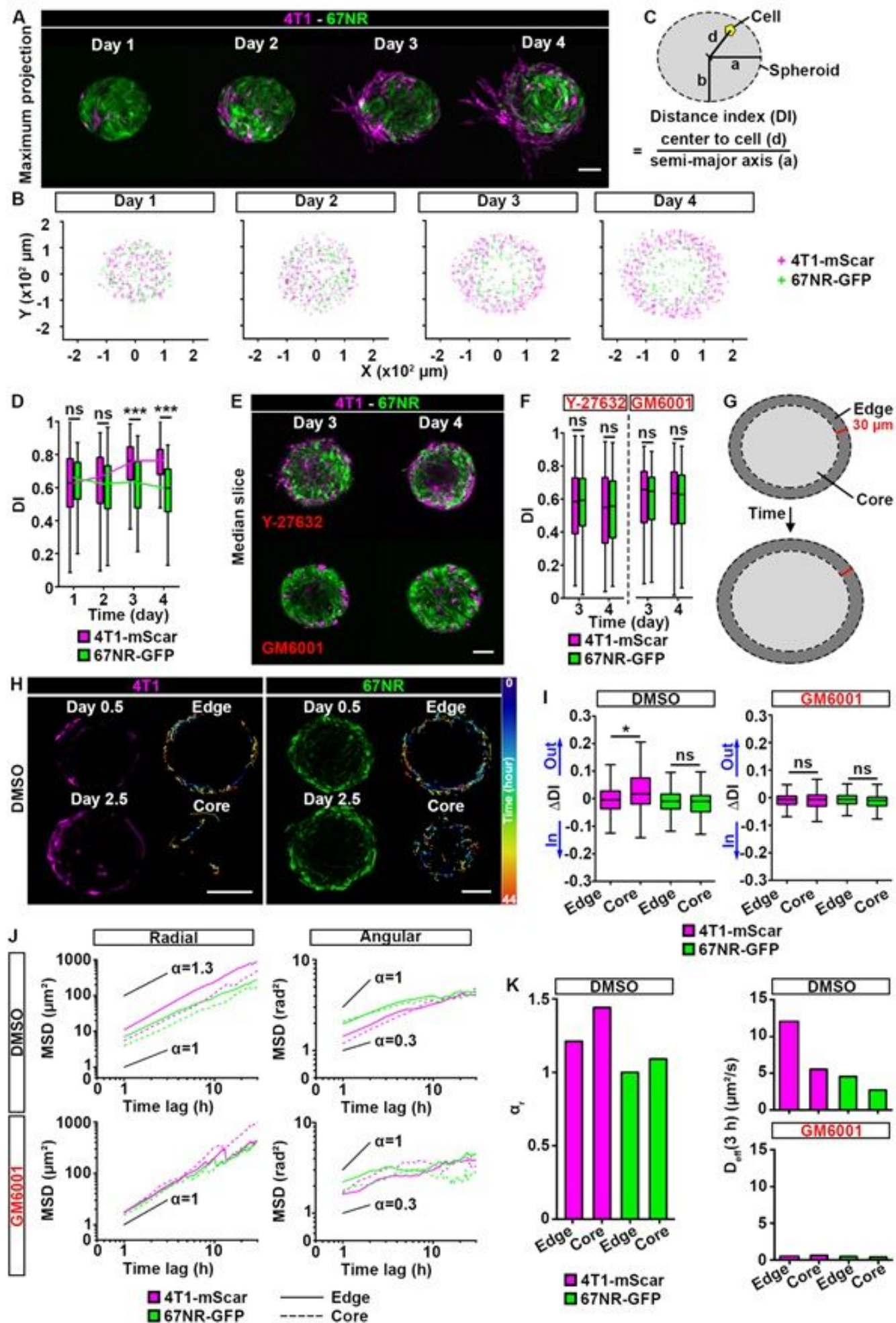

口4T1-mScar

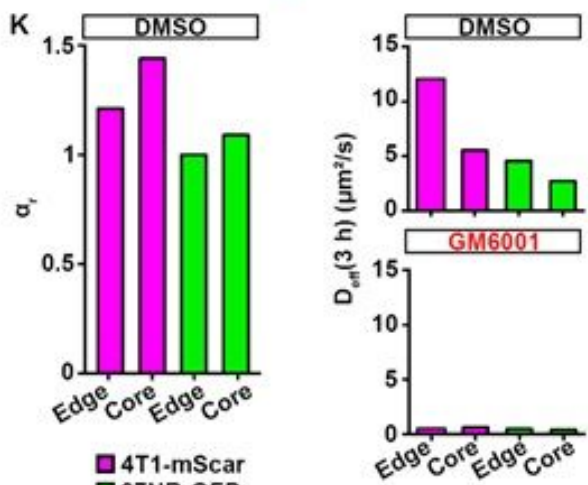

口4T1-mScar 口67NR-GFP

口67NR-GFP

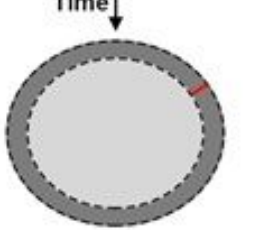


representation of the distance index (DI). b represents the semi-minor axis. (D) Quantification of the Distance Index (Dl; relative distance of each cell to spheroid center, see Materials and Methods) for 4T1mScarlet (4T1-mScar, magenta boxes) and 67NR-GFP (green boxes) cells from (A, B). P=0.775, 0.0983, $1.32 \times 10-14$ and $<2.20 \times 10-16$ were obtained by the Wilcoxon rank sum test. (E) Representative micrographs (median slice) of mixed spheroids cultured in a 3D collagen I matrix for 3 and 4 days. Spheroids were treated from day 0 with an inhibitor of cell contractility (ROCK inhibitor, Y-27632, top panels) or a pan-MMP inhibitor (GM6001, bottom panels). Scale bar: $100 \mu \mathrm{m}$. (F) Quantification of the distance index (DI) for 4T1-mScarlet (4T1-mScar, magenta boxes) and 67NR-GFP (green boxes) cells from (E). $P=0.569,0.929,0.282$ and 0.428 were obtained by the Wilcoxon rank sum test. (G) Schematic representation of the edge (dark gray) and core (light gray) compartments in a spheroid. $(\mathrm{H})$ Representative snapshot micrographs (single slice) of a mixed spheroid cultured in a 3D collagen I matrix. The time-lapse started $10 \mathrm{~h}$ (time $0 \mathrm{~h}$ ) post embedding and lasted for $44 \mathrm{~h}$ (time $44 \mathrm{~h}$ ). For 4T1mScarlet and 67NR-GFP cells, representative cell trajectories in the edge and core compartments are shown (right panels). The trajectories are color-coded according to time. Scale bars: $100 \mu \mathrm{m}$. (I)

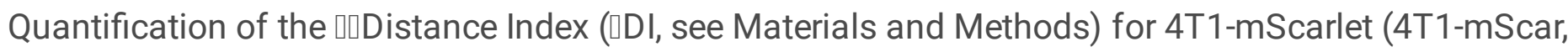
magenta boxes) and 67NR-GFP (green boxes) cells from (H). A positive $\mathrm{QDI}$ indicates cell motility towards the spheroid edge ("Out"), and a negative UDI indicates movement towards the spheroid center ("In"). Spheroids were treated from day 0 with DMSO control (left panel) or a pan-MMP inhibitor (GM6001, right panel). $P=0.0204,0.747,0.844$ and 0.107 were obtained by the Wilcoxon rank sum test. $(\mathrm{J})$ Mean square displacements (MSDs) for 4T1-mScarlet (4T1-mScar, magenta lines) and 67NR-GFP (green lines) cells from $(H)$. MSDs were calculated in the radial (left panels) and angular (right panels) directions of the polar coordinate system (see Materials and Methods), for edge (solid lines) and core (dashed lines) cells in spheroids treated with DMSO (top panels) and GM6001 (bottom panels), respectively. The plots are shown in log-log scale, highlighting the super-diffusive $(a>1)$, diffusive $(a=1)$, and sub-diffusive $(a<1)$ nature of motility. Solid lines serve as guides to the eye, indicating the average slopes (a values) corresponding to these different motility modalities (see Materials and Methods for details). (K) Power law exponent ar is shown (left panel) for 4T1-mScarlet (4T1-mScar, magenta bars) and 67NR-GFP (green bars) cells from edge and core compartments in a spheroid. Effective diffusion coefficient in radial direction (see Materials and Methods for details) is shown for DMSO control (right, top panel) or a panMMP inhibitor (GM6001, right, bottom panel) from edge and core compartments in a spheroid. All data are represented as boxplots with median (line), 25th/75th percentiles (boxes) and maximum/minimum (whiskers). Statistical significance was defined as ${ }^{*} p<0.05$, ${ }^{*} p<0.01,{ }^{* *} p<0.001$. Additional information on the metrics and statistics can be found in the Table S1. 

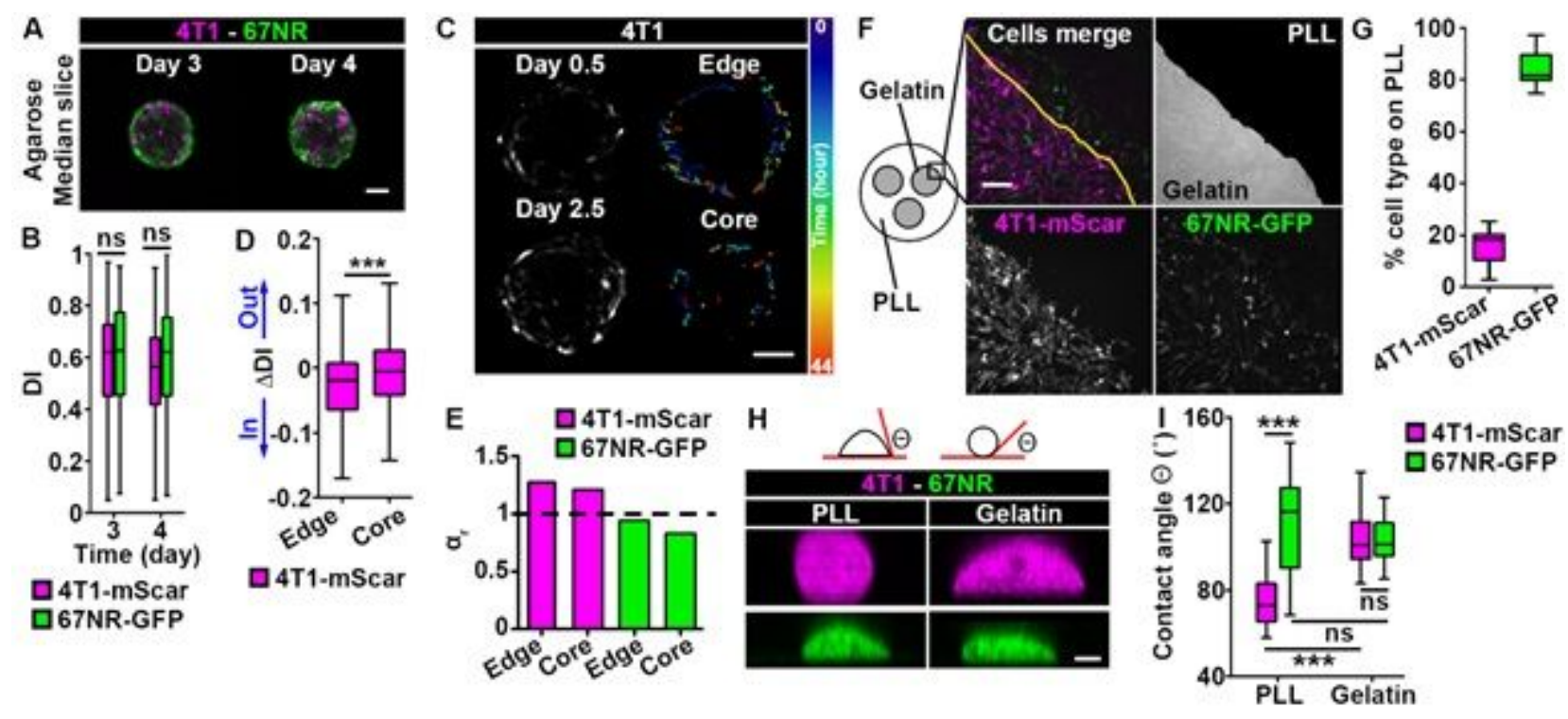

\section{Figure 3}

$4 T 1$, but not $67 N R$, cells are sensitive to the presence of adhesive ECM. (A) Representative micrographs (median slice) of mixed spheroids cultured in an agarose matrix for 3 or 4 days. Scale bar: $100 \mu \mathrm{m}$. (B) Quantification of the distance index (DI) for 4T1-mScarlet (4T1-mScar, magenta boxes) and 67NR-GFP (green boxes) cells from (A). $P=0.322$ and 0.999 were obtained by the Wilcoxon rank sum test. (C) Representative snapshot micrographs (single slice) of a mixed spheroid cultured in a 3D collagen I matrix. Only 4T1-mScarlet cells are shown. The time-lapse started $10 \mathrm{~h}$ (time $0 \mathrm{~h}$ ) post embedding and lasted for $44 \mathrm{~h}$ (time $44 \mathrm{~h}$ ). Representative cell trajectories in the edge and core compartments are shown (right panels). The trajectories are color-coded according to time. Scale bar: $100 \mu \mathrm{m}$. (D) Quantification of the $\mathrm{D}$ distance index (DDI) for 4T1-mScarlet cells from (C). A positive DDI indicates cell motility towards the spheroid edge ("Out"), and a negative DDI indicates movement towards the spheroid center ("In"). $P=0.000750$ was obtained by the Wilcoxon rank sum test. (E) Power law exponent ar is shown for 4T1mScarlet (4T1-mScar, magenta bars) and 67NR-GFP (green bars) cells from edge and core compartments in a spheroid. (F) Schematic representation of the 2D cell-ECM competition assay and representative micrographs of cells present at the gelatin/poly-L-lysine (PLL) interface $24 \mathrm{~h}$ post plating (top, right panel). 4T1-mScarlet (4T1-mScar; bottom, left panel) and 67NR-GFP (bottom, right panel) cells were plated on the gelatin islands only. Scale bar: $200 \mu \mathrm{m}$. (G) Quantification of the percentage of 4T1mScarlet (4T1-mScar, magenta boxes) and 67NR-GFP (green boxes) cells on poly-L-lysine (PLL) from (F). $(H)$ Schematic representation of the contact angle $(q)$ and representative micrographs (orthogonal views) of 4T1-mScarlet and 67NR-GFP cells on poly-L-lysine (PLL) or gelatin, $5 \mathrm{~h}$ post plating. Scale bar: $5 \mu \mathrm{m}$. (I) Quantification of the contact angle $q$ for cells from $(H)$. PLL, 4T1 vs. 67NR: $P=1.25 \times 10-9$; gelatin, 4T1 vs. 67NR: $P=0.563 ; 4 T 1$, PLL vs. gelatin: $P=4.13 \times 10-10$ and 67NR, PLL vs. gelatin: $P=0.0571$ were obtained by the Wilcoxon rank sum test. All data are represented as boxplots with median (line), 25th/75th percentiles (boxes) and maximum/minimum (whiskers). Statistical significance was defined as ${ }^{*} p<0.05,{ }^{*} p<0.01,{ }^{* *} p<0.001$. Additional information on the metrics and statistics can be found in the Table S1. 


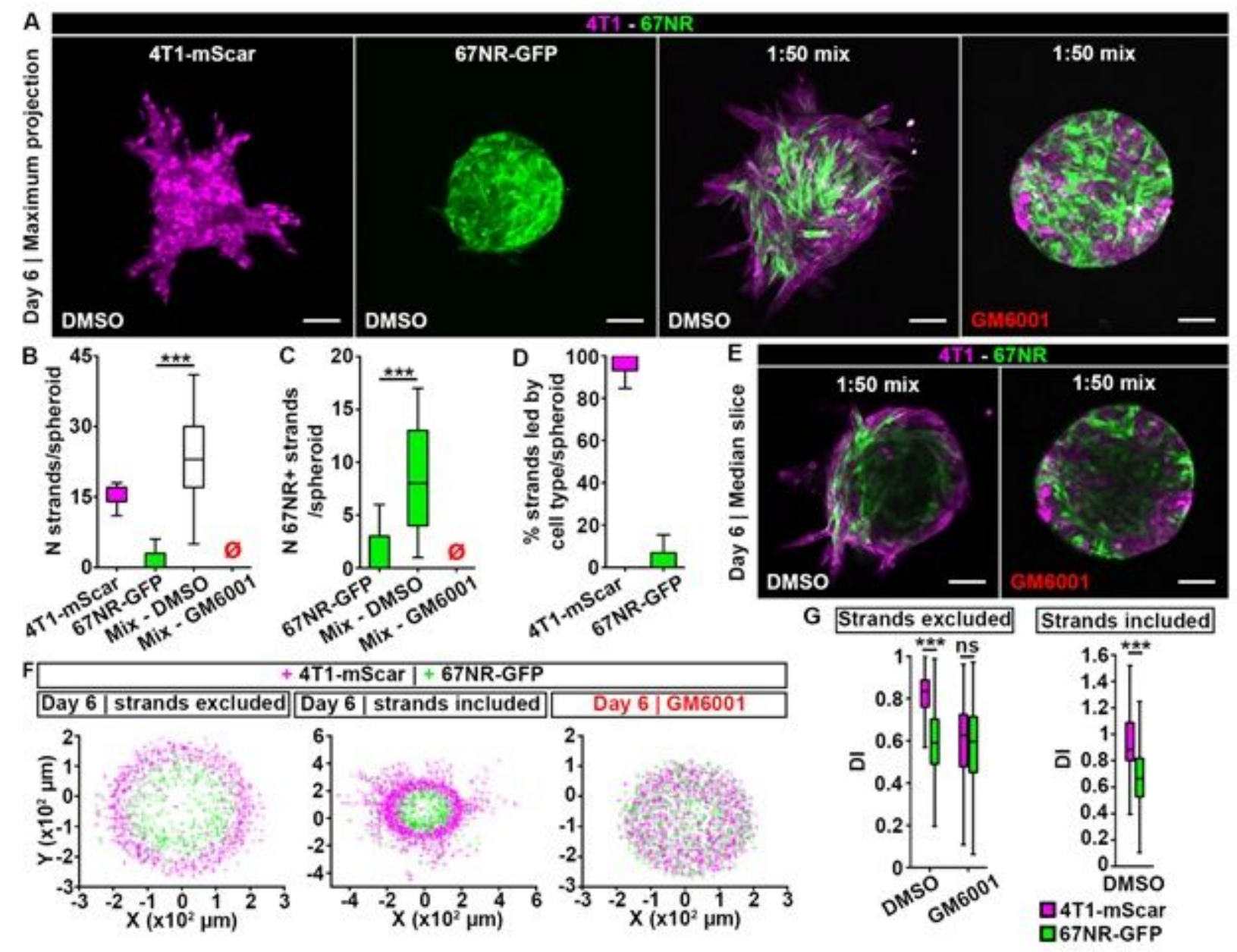

Figure 4

4T1 cells lead 67NR cells in an MMP-dependent cooperative invasion. (A) Representative micrographs (maximum projection) of spheroids made of a single or mixed 4T1-mScarlet and 67NR-GFP cell line, cultured in a 3D collagen I matrix for 6 days. Mixed spheroids were made at a 1 to 50 ratio of 4T1mScarlet to 67NR-GFP cells. Spheroids composed of single cell lines were made in accordance with this ratio. Spheroids were treated from day 0 with a pan-MMP inhibitor (GM6001, right panel) or DMSO control. Scale bars: $100 \mu \mathrm{m}$. (B-D) Quantification of the number of strands per spheroid (B), the number of strands containing 67NR cells (67NR+) per spheroid (C), and the percent of strands led by 4T1-mScarlet (4T1-mScar, magenta box) or 67NR-GFP (green boxes) cells (D) for single or mixed (white box) spheroids from (A). The red empty symbols indicate zero values. $P=1.90 \times 10-7$ (B) and $9.86 \times 10-6$ (C) were obtained by the Wilcoxon rank sum test. (E) Representative micrographs (median slice) of mixed spheroids from (A). Scale bars: $100 \mu \mathrm{m}$. (F) Coordinates of 4T1-mScarlet (4T1-mScar, magenta crosses) and 67NR-GFP (green crosses) cells from mixed spheroids in $(A)$ and $(E)$. Cells present in strands were excluded (left panel) or included (middle panel) in the analysis. (G) Quantification of the distance index (DI) for 4T1mScarlet (magenta boxes) and 67NR-GFP (green boxes) cells from (E, F). P<2.20x10-16, 0.0665 and $<2.20 \times 10-16$ were obtained by the Wilcoxon rank sum test. All data are represented as boxplots with median (line), 25th/75th percentiles (boxes) and maximum/minimum (whiskers). Statistical significance 
was defined as ${ }^{*} p<0.05,{ }^{*} p<0.01$ and ${ }^{* *} p<0.001$. Additional information on the metrics and statistics can be found in the Table S1.

A

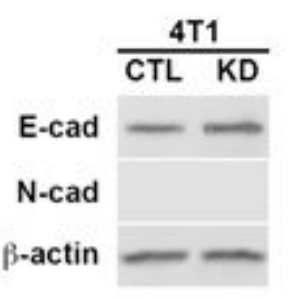

$B$
$\stackrel{5}{5}$
$\stackrel{5}{5}$<smiles>[Mg]O[Mg][Mg]</smiles>
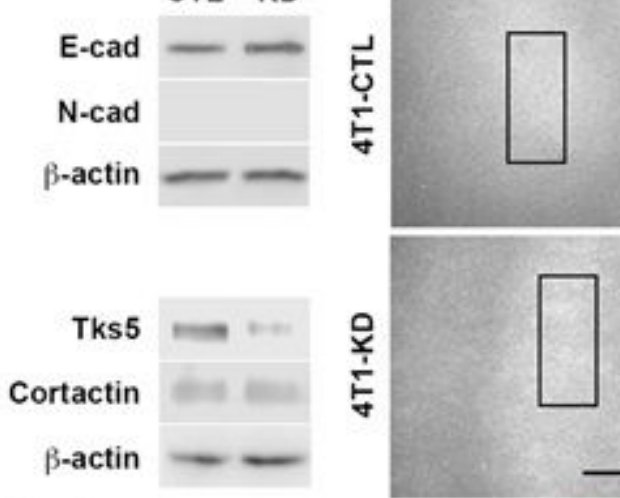

D
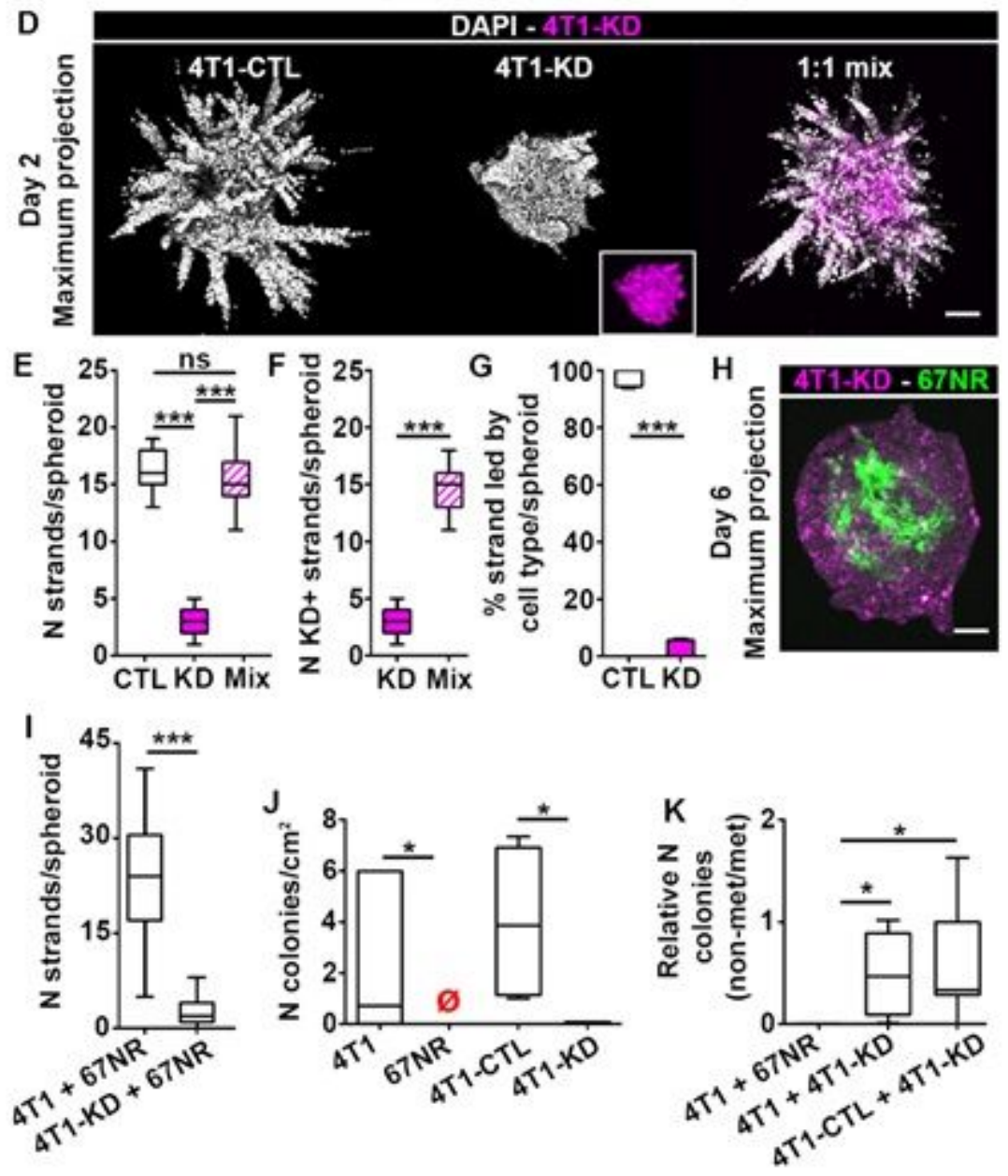

\section{Figure 5}

Mixture of cells with and without invadopodia exhibit cooperation in invasion and lung metastasis. (A) Western blot analysis of E/N-cadherin, Tks5 and cortactin expression levels in 4T1-shControl (4T1-CTL) and 4T1-mScarlet-mTks5 KD (4T1-KD) cells. $\beta$-actin is used as a loading control. (B) Representative micrographs of gelatin degradation for 4T1-shControl (4T1-CTL, top panels) and 4T1-mScarlet-mTks5 KD (4T1-KD, bottom panels) cells $18 \mathrm{~h}$ after plating. The inserts show a $2 \mathrm{X}$ zoom-in of the boxed area and 
arrowheads indicate representative degradation holes. Scale bar: $20 \mu \mathrm{m}$. (C) Quantification of the degradation area per cell for 4T1-shControl (4T1-CTL) and 4T1-mScarlet-mTks5 KD (4T1-KD) cells from (B). $P=7.24 \times 10-14$ was obtained by the Wilcoxon rank sum test. (D) Representative micrographs (maximum projection) of spheroids made of a single or mixed 4T1-shControl (4T1-CTL) and 4T1mScarlet-mTks5 KD (4T1-KD) cell lines, cultured in a 3D collagen I matrix for 2 days. Mixed spheroids were made at a 1 to 1 ratio. Scale bar: $100 \mu \mathrm{m}$. (E-G) Quantification of the number of strands per spheroid $(E)$, the number of strands containing 4T1-mScarlet-mTks5 KD cells (KD+) per spheroid (F), and the percentage of strands led by 4T1-shControl (4T1-CTL) or 4T1-mScarlet-mTks5 KD (4T1-KD) cells (G) for single or mixed spheroids from (D). $P<2.20 \times 10-16$ was obtained by the two-sample t-test, $P=7.26 \times 10-7$ and 0.380 were obtained by the Welch two-sample t-test in $(E) . P=8.49 \times 10-7$ was obtained by the Welch two-sample t-test in (F). (H) Representative micrographs (maximum projection) of a mixed 4T1-mScarletmTks5 KD (4T1-KD) and 67NR-GFP spheroid, cultured in a 3D collagen I matrix for 6 days. Mixed spheroids were made at a 1 to 50 ratio. Scale bar: $100 \mu \mathrm{m}$. (I) Quantification of the number of strands per spheroid for mixed spheroids from Fig. 4A (4T1+67NR) and (H) (4T1-KD+67NR). $P=3.36 \times 10-6$ was obtained by the Wilcoxon rank sum test. $(J)$ Quantification of the number of colonies per $\mathrm{cm} 2$ of culture dishes from the lung clonogenic assay. Mice were inoculated with a single (left panel) or mixed (right panel) 4T1, 67NR, 4T1-shControl (4T1-CTL) and 4T1-mScarlet-mTks5 KD (4T1-KD) cell lines. (K) Lung clonogenic assay in mice inoculated with cell mixtures. Relative numbers of colonies from nonmetastatic cells over metastatic cells was reported. $P=0.0104,0.0323,0.0250$ and 0.0262 were obtained by the Wilcoxon rank sum test. All data are represented as boxplots with median (line), 25th/75th percentiles (boxes) and maximum/minimum (whiskers). Statistical significance was defined as ${ }^{*} \mathrm{p}<0.05$, ${ }^{* \star} p<0.01$ and ${ }^{* \star *} p<0.001$. Additional information on the metrics and statistics can be found in the Table S1.

\section{Supplementary Files}

This is a list of supplementary files associated with this preprint. Click to download.

- MovieS1.avi

- MovieS2.avi

- MovieS3.avi

- MovieS4.avi

- MovieS5.avi

- MovieS6.avi

- MovieS7.avi

- Movies8.avi

- Movies9.avi

- MovieS10.avi 
- SupplementaryMaterialsLPerrinetal0615.docx 